\title{
Supramaximal cholecystokinin displaces Munc18c from the pancreatic acinar basal surface, redirecting apical exocytosis to the basal membrane
}

\author{
Herbert Y. Gaisano, ${ }^{1,2}$ Manfred P. Lutz, ${ }^{3}$ Juergen Leser, ${ }^{3}$ Laura Sheu, ${ }^{1}$ Grit Lynch, ${ }^{3}$ \\ Lan Tang, ${ }^{1}$ Yoshikazu Tamori, ${ }^{4}$ William S. Trimble, ${ }^{2,5}$ and Anne Marie F. Salapatek ${ }^{2}$
}

${ }^{1}$ Department of Medicine, and

${ }^{2}$ Department of Physiology, University of Toronto, Toronto, Ontario, Canada

${ }^{3}$ University of Ulm, Department of Medicine, Ulm, Germany

${ }^{4}$ Kobe University School of Medicine, Kobe, Japan

${ }^{5}$ Department of Biochemistry, University of Toronto, Toronto, Ontario, Canada, and Cell Biology Program

of the Hospital for Sick Children of Toronto, Toronto, Ontario, Canada

Address correspondence to: Herbert Y. Gaisano, Room 7226 Medical Science Building, University of Toronto, Toronto, Ontario, Canada M5S 1A8. Phone: (416) 978-1526; Fax: (416) 978-8765; E-mail: herbert.gaisano@utoronto.ca.

Herbert Y. Gaisano and Manfred P. Lutz contributed equally to this work.

Received for publication December 10, 1999, and accepted in revised form October 8, 2001.

\begin{abstract}
Exocytosis at the apical surface of pancreatic acinar cells occurs in the presence of physiological concentrations of cholecystokinin (CCK) but is inhibited at high concentrations. Here we show that Munc18c is localized predominantly to the basal membranes of acinar cells. Supramaximal but not submaximal CCK stimulation caused Munc18c to dissociate from the plasma membrane, and this displacement was blocked by protein kinase $\mathrm{C}$ (PKC) inhibitors. Conversely, whereas the CCK analog CCK-OPE alone failed to displace Munc18c from the membrane, this agent caused Munc18c displacement following minimal PKC activation. To determine the physiological significance of this displacement, we used the fluorescent dye FM1-43 to visualize individual exocytosis events in real-time from rat acinar cells in culture. We showed that supramaximal CCK inhibition of secretion resulted from impaired apical secretion and a redirection of exocytic events to restricted basal membrane sites. In contrast, CCK-OPE evoked apical exocytosis and could only induce basolateral exocytosis following activation of PKC. Infusion of supraphysiological concentrations of CCK in rats, a treatment that induced tissue changes reminiscent of mild acute pancreatitis, likewise resulted in rapid displacement of Munc18c from the basal membrane in vivo.
\end{abstract}

J. Clin. Invest. 108:1597-1611 (2001). DOI:10.1172/JCI200109110.

\section{Introduction}

The pancreatic acinar cell synthesizes and releases large amounts of zymogen enzymes (1) necessary for digestion. These enzymes are packaged into large dense-cored zymogen granules (ZGs), and these ZGs are stored and packed into the apical pole of the acinar cell where they occupy as much as $30 \%$ of the cell volume $(1,2)$. Considering that exocytosis occurs only on the apical membrane surface that is limited to less than $10 \%$ of the total membrane surface area (2), regulated exocytosis in the acinar cell must be efficient and tightly controlled. However, at supraphysiologic (or supramaximal) concentrations of the secretagogue cholecystokinin $(\mathrm{CCK} ;>100 \mathrm{pM})$, secretion is inhibited (although still near $70 \%$ of maximal levels) (3). Ultrastructural studies have suggested that supramaximal CCK may totally block apical exocytosis, yet by this method, only very rare exocytosis events were observed at the lateral plasma membrane (4). It is therefore unclear how the substantial enzyme secretion could occur during supramaximal CCK stimulation. Interestingly, high-dose CCK infusion into rats results in a mild form of acute pancreatitis (4-6) and has been the best established model of clinical interstitial pancreatitis (7). The cellular events observed in this experimental pancreatitis model remarkably resemble those observed with supramaximal CCK of dispersed rat pancreatic acini, including the anomalies of ZG secretion (4).

A CCK analog called CCK-OPE evokes secretion at low concentrations (8-11) but does not exhibit the supramaximal inhibition of secretion at high doses $(>0.1 \mu \mathrm{M}$ CCK-OPE) $(8,9)$. We previously reported that while CCK is coupled to protein kinase C (PKC) activation, CCK-OPE is only weakly capable of activating $\operatorname{PKC}(8,9,11)$. Moreover, minimal activation of PKC by nanomolar concentrations of phorbol ester (i.e., $1 \mathrm{nM}$ 12-0-tetradecanoylphorbolacetate (TPA)) can induce supramaximal CCK-OPE to mimic the actions of supramaximal CCK $(9,10)$, suggesting that these effects are caused by PKC activation.

Precise temporal and spatial control of ZG secretion is likely to involve the regulation of the SNARE machinery responsible for exocytosis. Originally identified in neurons as proteins necessary for regulated neurotransmitter release $(11,12)$, specialized isoforms of proteins have 
been found in all cell types and at specific cellular compartments where they likely mediate each step in membrane trafficking. A simplified view of the SNARE hypothesis, which explains their functions, would be that membrane fusion is driven by the formation of a complex between a donor vesicle (v-) SNARE protein from the VAMP family and target ( $\mathrm{t}-)$ membrane SNAREs of the syntaxin and SNAP-25 families (13). A corollary hypothesis is that additional proteins are required to prevent these cognate $\mathrm{v}$ - and $\mathrm{t}$-SNAREs from coming together inappropriately $(14,15)$. In neurons, Munc18a (also called nSec1) binds to syntaxin-1 with very high affinity and blocks the ability of syntaxin- 1 to bind to VAMP-2 and SNAP-25 in vitro (14). PKC phosphorylation of Munc18a inhibits its binding to syntaxin $(16,17)$, and its dissociation from syntaxin allows binding VAMP-2 and SNAP-25 (14). One role of Munc18 may therefore be as an inhibitor of syntaxin interactions with SNAP-25 and VAMP, and this could be released by PKC phosphorylation $(16,17)$. We have previously reported the SNARE isoforms of VAMP (VAMP-2 and -3) $(18,19)$, SNAP-25 (SNAP-23) $(20)$, and syntaxin (syntaxin-2 through -4) (21) expressed in the pancreatic acinar cell. In these cells, syntaxin-2 is restricted to the apical membrane, syntaxin- 3 to the granule membrane, syntaxin- 4 to the basolateral membrane, SNAP- 23 to the basolateral membrane, and VAMP-2 to the granule. Thus, in the acinar cell, the basolateral plasma membrane possesses a complete set of known t-SNAREs (SNAP-23 and syntaxin-4) capable of binding to the vSNARE VAMP-2 to form a SNARE complex. In vitro studies of recombinant proteins indeed demonstrated stable binding of VAMP-2, SNAP-23, and syntaxin-4 (22), raising the possibility that a complex of VAMP-2/ SNAP-23/syntaxin-4 could mediate basolateral exocytosis of digestive enzymes within the pancreas. Non-neuronal Munc18 isoforms were also identified, including Munc18b and Munc18c (21-26). Munc18c binds to syntaxin- 4 and blocks its binding to SNAP-23 and VAMP-2 $(24,25)$, and overexpression of Munc18c in adipocytes inhibits insulin-mediated GLUT-4 translocation to the plasma membrane and glucose transport (26).

We hypothesize that alterations in ZG secretion may involve changes in SNARE protein regulation following supramaximal CCK stimulation. To examine this in more detail we determined the subcellular distribution of Munc18c and monitored the timing and location of exocytosis in vitro and in vivo in untreated or supramaximally stimulated acini. Our results suggest that supramaximal CCK activates PKC, leading to displacement of Munc18c and a shift in exocytosis from the apical to the basal surface. This redirected secretion would lead to interstitial secretion that could contribute to the development of pancreatitis.

\section{Methods}

Antibodies and reagents. Antibodies generated against Munc18c $(25,26)$, SNAP-23 (20), and VAMP-2 $(18,19)$ were previously described. Syntaxin-4 antibody was a gift from J. Pessin (University of Iowa, Iowa City, Iowa, USA). CCK-8, cerulein, and CCK-OPE were from Research Plus (Bayonne, New Jersey, USA). Calphostin C was from Calbiochem-Novabiochem Corp. (San Diego, California, USA), and FM1-43 was from Molecular Probes Inc. (Eugene, Oregon, USA).

Dispersed acini and subcellular fractionation and immunoblotting. The preparation of isolated rat acini was performed by a mechanical and enzymatic dissociation technique as we have previously described (8-11). The acini were resuspended in oxygenated Krebs-RingerHEPES buffer, consisting of (in mM): $104 \mathrm{NaCl}, 5 \mathrm{KCl}$, $1 \mathrm{KH}_{2} \mathrm{PO}_{4}, 1.2 \mathrm{MgCl}_{2}, 2 \mathrm{CaCl}_{2}, 0.2 \%$ (wt/vol) BSA, $0.01 \%$ (wt/vol) soybean trypsin inhibitor, $10 \mathrm{mM}$ glucose, 25 mM HEPES/NaOH, pH 7.4, supplemented with MEM and glutamine. The acini were then stimulated with agonists as indicated and examined by confocal microscopy as described below.

Subcellular fractionation. The dispersed pancreatic acini were subjected to the indicated stimulation protocols. The acini were then pelleted by low-speed centrifugation $\left(300 \mathrm{~g}, 4^{\circ} \mathrm{C}\right)$ and homogenized with a Teflon homogenizer, followed by a 20 -minute ultracentrifugation $(150,000 \mathrm{~g})$ to separate the crude membranes from the cytosolic supernatant fraction. Highly purified plasma membranes and ZG membranes were prepared as previously described by sucrose density gradient centrifugation (18-21).

Immunoblotting. Immunoblotting was performed as previously described (18-21). Briefly, equal amounts of protein were separated by denaturing SDS-PAGE and transferred to PVDF membranes (Immobilon-P; Millipore Corp., Bedford, Massachusetts, USA), which were then blocked (50 mM Tris- $\mathrm{HCl}, \mathrm{pH} 7.8,100 \mathrm{mM} \mathrm{NaCl}$, $0.5 \%$ Tween-20, $2 \%$ [wt/vol] BSA) overnight. These membranes were immunodecorated with primary antibodies, and the respective antigen-antibody complexes were identified by appropriate peroxidase-conjugated secondary antibodies and enhanced chemiluminescence (ECL; Amersham, Arlington Heights, Illinois, USA) followed by visualization by exposure to Kodak X-OMAT AR films (Eastman Kodak, Rochester, New York, USA). Equal protein loading was verified by India ink staining of blotting membranes.

Immunoprecipitation. Immunoprecipitation was performed as we had previously reported $(21,25,26)$. Five to ten micrograms of Munc18c antibody was coupled to protein $\mathrm{G}$ sepharose (Pharmacia Biotech, Buckinghamshire, United Kingdom) beads, and incubated with solubilized pancreatic acinar plasma membranes $(0.5-1$ $\mathrm{mg})$ in lysis buffer (50 mM Tris/ $\mathrm{HCl}$ [pH 7.4], $150 \mathrm{mM}$ $\mathrm{NaCl}, 1 \mathrm{mM} \mathrm{MgCl}_{2}, 1 \mathrm{mM} \mathrm{CaCl}_{2}$, and $1 \%$ Triton X-100 with $1 \mathrm{mM}$ PMSF, $10 \mathrm{mM}$ benzamidine, $1 \mathrm{mM}$ leupeptin, $1 \mu \mathrm{M}$ pepstatin, and $0.5 \mathrm{mg} / \mathrm{ml}$ soybean trypsin inhibitor) for 3 hours at $4^{\circ} \mathrm{C}$. The proteins bound to the beads were then eluted and separated on a $10 \%$ SDS-PAGE. These proteins (Munc18c) and coimmunoprecipitated proteins (syntaxin-4) were then identified by immunoblotting. 
Confocal immunofluorescence microscopy. Confocal immunofluorescence microscopy was done as previously described (19-21). Dispersed acini or 5- $\mu \mathrm{m}$-thick sections of the treated rat pancreas were plated on glass coverslips and fixed with $2 \%$ paraformaldehyde, followed by rinsing in PBS for 5 minutes. These tissues on the coverslips were then blocked with $5 \%$ normal goat serum with $0.1 \%$ saponin for 0.5 hours at room temperature, and washed and incubated with the indicated primary antibodies for 1 hour at 1:100 dilution. This was followed by incubation with appropriate fluorochrome-conjugated secondary antisera (1 hour, $25^{\circ} \mathrm{C}$ ), along with colabeling with FITC-conjugated phalloidin (0.2 $\mu \mathrm{M}$; Molecular Probes Inc.). The coverslips were then mounted on slides in a fading retarder$0.1 \%$, phenylenedediamine in glycerol, and examined using a laser scanning confocal imaging system (Carl Zeiss, Oberkochen, Germany). Imaging was done at the same fluorescence gain intensity and with minimal to no bleaching to allow direct comparison of the studies. Analysis of the confocal fluorescent images was by $\mathrm{NIH}$ Image (NIH, Bethesda, Maryland, USA) and Excel (Microsoft, Seattle, Washington, USA) software.

A 3D image was generated by having the serial $x y$ images first processed with a $3 \times 3$ pixel median filter (Scananalytics, Fairfax, Virginia, USA) and then 3Dreconstructed with a volume-rendering software (VoxBlast; VayTek, Fairfield, Iowa, USA).

Colocalization of FM1-43 and amylase in nonpermeabilized acini. This was performed as similarly described for the lactotroph study reported by Angleson et al. $(27,28)$. Pancreatic acini plated on glass coverslips were stimulated with $10 \mathrm{nM} \mathrm{CCK}$ for $15-20$ minutes $\left(37^{\circ} \mathrm{C}\right)$ and not washed nor permeabilized. Rabbit anti-amylase antibodies (1:1000; Sigma Chemical Co., St. Louis, Missouri, USA) were then added for 20 minutes $\left(22^{\circ} \mathrm{C}\right)$ followed by a mild wash, and incubated with $\mathrm{Cy}-5-$ labeled goat anti-rabbit secondary antibodies (1:400; Jackson Laboratory, West Grove, Pennsylvania, USA) for 20 minutes $\left(22^{\circ} \mathrm{C}\right)$ along with $4 \mu \mathrm{M}$ FM1-43. The acini were then washed twice with FM1-43-containing media and imaged by confocal microscopy for FM1-43 fluorescence (as above), and for $\mathrm{Cy}-5$ fluorescence with a 640/20-nm excitation and 680/15-nm emission filters. FM1-43 imaging of exocytosis. Dispersed acinar cells in BSA-free media were plated on glass coverslips and mounted in a heated chamber kept at $37^{\circ} \mathrm{C}$. The cells were then incubated with $4 \mu \mathrm{M}$ FM1-43 for 15 minutes at $37^{\circ} \mathrm{C}$ prior to the start of the experiment, until we were able to observe a stable low or basal level of fluorescence staining that is uniformly distributed along the plasma membrane of all the acinar cells. The FM143 was maintained in the extracellular media for the entire duration of the experiment. Imaging of the cells was performed using two imaging systems. The first system is an epifluorescence imaging system to track real-time exocytic events (29) occurring at different regions of the acinar cells. This imaging system is comprised of an Olympus IX70 inverted epifluorescence microscope (Olympus America, New York, New York, USA), an Ultrapix camera with Kodak KAF1400 chip (Eastman Kodak) $(6.7 \times 6.7 \mu \mathrm{m}$ physical pixels, giving $67 \mathrm{~nm}$ per image pixel with a $100 \times$ oil immersion objective), and a personal computer with Merlin Imaging software (Olympus America). Fluorescence images of FM1-43-stained cells were obtained with 490/20-nm excitation provided by a monochromator (Olympus America) and 617/73-nm emission filters. Exposures lasted 0.4 seconds, and images were acquired at about $1 \mathrm{~Hz}$. Quantitative analyses were performed on raw images. Epifluorescent images were analyzed using Merlin software. Fluorescence of individual spots was measured in the manner described by Angleson and colleagues $(27,28)$. The mean pixel value inside a chosen "region of interest" circle centered around the "hotspot" (usual circle diameter, seven pixels) was normalized to the mean surface membrane fluorescence inside the circle at the same location measured for at least five frames immediately before the hotspot appearance. Intensity values were obtained for the average brightness attained across regions of interest. A brightness of 0 means the spot was the same brightness as the plasma membrane, and 1.0 means it was twice as bright. All data are reported as mean \pm SEM.

For confocal imaging we used a Carl Zeiss confocal microscope system equipped with LSM software (version 3.98; Carl Zeiss, Oberkochen, Germany) (18-21). The top and bottom of the acinus were identified and confocal sections were obtained at $1 \mu \mathrm{m}$ thickness, unless otherwise specified, in the $z$ axis at the indicated time intervals.

Supramaximal cerulein-induced rat pancreatitis. This was performed as we have previously described $(30,31)$. Male Wistar rats (200-250 g) were equipped with an intravenous cannula, followed by the indicated infusion protocols. Alternatively, drugs where given by intraperitoneal injection in 50-100 $\mu \mathrm{l}$ of PBS. At the indicated time points, the rats were anesthetized using ether and the ascending aorta was cannulated. After collection of blood (for amylase determination), animals were rapidly perfused with PBS followed by $4 \%$ formaldehyde in PBS and were then sacrificed. The pancreas was rapidly removed, postfixed for 2 hours, and transferred to PBS. This was then embedded in Tissue-Tek OCT (Sakura Finetek, Torrance, California, USA) and $5-\mu \mathrm{m}$ cryostat sections obtained and plated onto glass coverslips for confocal immunofluorescence microscopy studies described above. Parallel tissue sections from these treated rat pancreata were subjected to histology examination (hematoxylin and eosin staining) as indicated.

\section{Results}

Munc18c is localized to the basal plasma membrane of pancreatic acinar cells. In order to examine whether Munc18 isoforms contributed to the polarity of $Z G$ secretion in the acinar cell, we determined whether specific isoforms of Munc18 were expressed in acinar cells and their subcellular locations. Since Munc18c is the only isoform capable of binding syntaxin- 4 with high affinity $(25,26)$, we 

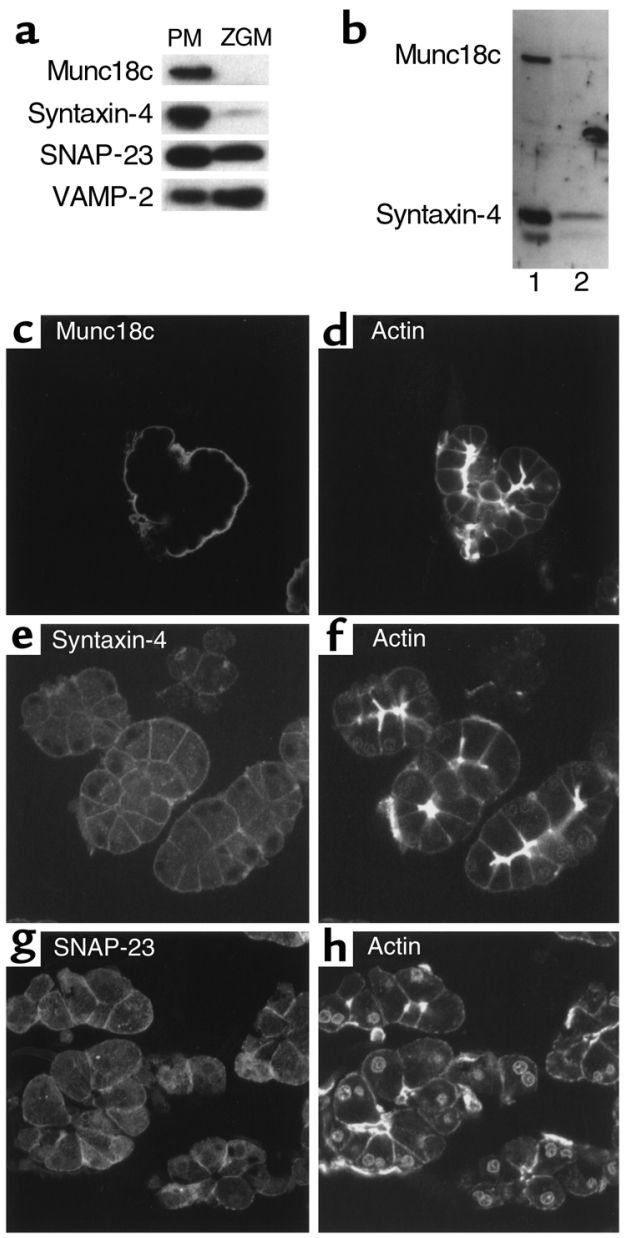

\section{Figure 1}

Localization of Munc18c, syntaxin-4, and SNAP-23 in dispersed rat pancreatic acinar cells. (a) Western blots of purified pancreatic acinar plasma membranes (PM) and ZG membranes (ZGM). Highly purified membranes (10 $\mu$ g protein) were separated on a $15 \%$ SDSPAGE and immunoblotted with anti-Munc18c, -syntaxin-4, -SNAP-23, and -VAMP-2 antibodies. (b) Immunoprecipitation of Munc18c from purified pancreatic acinar plasma membranes $(0.5 \mathrm{mg})$, and coimmunoprecipitation of syntaxin-4 (lane 1). Immunoprecipitation of total lysates (10-cm dish) of 3T3-L1 adipocytes were used as a positive control $(25,26)$ (lane 2$)$. (c-h) Laser confocal microscopy of dispersed acinar cells. Acinar cells were plated on coverslips and probed with (c) anti-Munc18c, (e) anti-syntaxin-4, or (g) anti-SNAP-23 antibodies, along with double-labeling with FITC-phalloidin in $\mathbf{d}, \mathbf{f}$, and $\mathbf{h}$, respectively, to indicate the plasma membrane staining and concentration of actin in the apical portion of the acinar cells.

examined the distribution of this protein in the acinar cell. To detect it we used a specific antibody generated against the C-terminal 14-amino acid sequence of mouse Munc18c $(25,26)$. This antibody detects a single $65-\mathrm{kDa}$ band (Figure 1a) in pancreatic plasma membranes isolated from unstimulated acini, but no Munc18c was detected in ZG membranes. Syntaxin-4, like Munc18c, was also almost exclusively present in the plasma membranes. SNAP-23 was also on the plasma membrane and was less abundant on ZG membranes, while VAMP-2 was more abundant in ZG membranes
$(18,19)$. Previous studies showed that overexpression of Munc18c prevents syntaxin-4 from binding to SNAP-23 (25) and VAMP-2 (24). Figure 1b shows that Munc18c coimmunoprecipitates with syntaxin-4 from purified pancreatic acinar plasma membranes, as it can from lysates of 3T3-L1 adipocytes (26), while SNAP-23 and VAMP-2 were absent from these complexes (data not shown). However, for these proteins to interact with each other in vivo, they would have to be located in the same subcellular compartment. We therefore compared the specific cellular location of Munc18c, syntaxin-4, and SNAP-23 in the pancreatic acinar cell. As shown in Figure $1 \mathrm{c}$, Munc18c is localized to the basal plasma membrane of freshly dispersed rat pancreatic acinar cells. In intact rat pancreatic tissues, we have also found a weak Munc18c signal on the lateral plasma membrane of the acinar cells (see Figure 8). This cellular location of Munc18c overlaps with the location of syntaxin-4 (Figure 1e) and SNAP-23 (Figure 1g). F-actin staining with phalloidin (Figure 1, d, f, and h) is used to define the apical membrane where the actin signal is strongest (30-32). Taken together, the basolateral plasma membrane of the acinar cell contains a set of t-SNARE proteins capable of forming a fusion complex with the ZG VAMP-2, and Munc18c may be responsible for preventing this complex from forming (15).

Supramaximal CCK stimulation displaces Munc18c from the basal plasma membrane. We next examined whether these proteins were coupled to secretagogue stimulation of the acinar cell. It is well established that CCK-8 dose-dependently stimulates secretion of amylase from dispersed rat pancreatic acini, which reaches maximal secretion at $100 \mathrm{pM}$, and at supramaximal concentrations (i.e., $10 \mathrm{nM}$ CCK-8) an inhibition of secretion from the maximal level is observed $(3,8)$. In contrast to CCK, CCK-OPE is as efficacious a secretagogue but does not exhibit an inhibition of secretion at supramaximal doses $(>0.1 \mu \mathrm{M})(8)$. We examined acini exposed to these CCK-8 stimulation protocols for the locations of Munc18c, syntaxin-4, and SNAP-23 by laser confocal microscopy. Stimulation of the acini by submaximal CCK-8 (30 pM; Figure 2a) for even as long as 1 hour had no effect on Munc18c cellular location. In contrast, supramaximal CCK-8 (10 nM; Figure $2 \mathrm{~b}$ ) stimulation for just 15 minutes caused Munc18c to dissociate from the basal plasma membrane into the cytosol, whereas supramaximal concentrations of CCK-OPE ( $1 \mu \mathrm{M}$; Figure $2 \mathrm{c})$ stimulation for as long as 1 hour had no effect on Munc18c cellular distribution. In contrast to Munc18c, syntaxin-4 (Figure 2h) and SNAP-23 (Figure 2i) were not displaced from the plasma membrane by supramaximal CCK-8 stimulation (30 minutes), and these signals appeared identical to unstimulated cells in Figure 1, e and g, respectively.

To examine the time course of the displacement of Munc18c from the plasma membrane into the cytosol, we fractionated into plasma membrane and cytosol fractions from the acini that were stimulated with 10 nM CCK- 8 at timed intervals of 1 minute to 15 min- 
utes. In Figure $2 \mathrm{j}$, a reduction of plasma membrane Munc18c levels can be detected after 5 minutes of 10 nM CCK-8 stimulation, and further reduction can be detected at 15 minutes of stimulation. In contrast, the cytosolic Munc18c levels increased at the earliest time point of 1 minute but, thereafter, actually decreased to a stable level below the control level. Of note, in the cytosol fraction, we discovered only one other major immunoreactive Munc18c protein, which is a smaller $\sim 35-\mathrm{kDa}$ protein that appeared at 1 minute. The levels of this cytosol 35-kDa Munc18c protein increased progressively up to 15 minutes of stimulation, which reciprocated the progressive reduction of the plasma membrane Munc18c levels. This 35-kDa cytosolic Munc18c protein is presumably a degraded product of the larger Munc18c protein that was displaced from the plasma membrane into the cytosol. In support, this $35-\mathrm{kDa}$ Munc18c protein was not present in the plasma membrane fractions and was almost undetectable in the cytosol at the basal state, but became abundant right after CCK stimulation. Both the membrane and cytosolic Munc18c proteins appeared as a doublet, which may be due to protein phosphorylation, and this was more often seen with the stimulated acini than with unstimulated acini (as in Figure 1a). The plasma membrane levels of syntaxin-4 and SNAP-23 (data not shown) were unchanged during the entire duration of 10 nM CCK-8 stimulation. Also, we did not notice a displacement of syntaxin-4 or SNAP-23 into the cytosolic fraction (data not shown).

Munc18c displacement from the basolateral plasma membrane is mediated by a PKC-dependent mechanism. We had previously reported that minimal PKC preactivation with low concentrations of TPA $(1 \mathrm{nM})$ followed by supramaximal concentrations of CCK-OPE (1 $\mu \mathrm{M})$ resulted in an inhibition of maximal secretion from pancreatic acinar cells, which mimicked the effects of supramaximal CCK-8 stimulation $(9,10)$. However, the precise mechanism for this secretory response remains unknown. Confocal microscopy revealed that $1 \mathrm{nM}$ TPA stimulation alone (for 30 minutes) had minimal effects on Munc18c plasma membrane location (Figure 2d), but exposure to micromolar TPA concentrations, which would maximally activate PKC and stimulate near-maximal enzyme secretion (9), caused Munc18c displacement (Figure 2e). Of note, when the acini were preincubated with $1 \mathrm{nM}$ TPA $\left(15\right.$ minutes, $\left.37^{\circ} \mathrm{C}\right)$ followed by supramaximal CCK-OPE $(1 \mu \mathrm{M}, 30$ minutes $)$ stimulation, Munc18c was also displaced from the plasma membrane (Figure $2 \mathrm{f}$ ) into the cytosol in a manner identical to the effects observed with supramaximal CCK-8 (Figure $2 \mathrm{~b}$ ) and $1 \mu \mathrm{M}$ TPA (Figure $2 \mathrm{e}$ ). These studies strongly suggest that Munc18c dissociation from the plasma membrane is a specific supramaximal CCK- and PKCmediated event. To confirm this, acini were preincubated with the specific PKC inhibitor calphostin C (500 nM, 40 minutes) followed by supramaximal CCK-8 (10 nM, 30 minutes, $37^{\circ} \mathrm{C}$ ) stimulation (Figure $2 \mathrm{~g}$ ). The PKC inhibitor was able to prevent supramaximal CCK-8 from causing Munc18c dissociation from the plasma membrane (compare Figure $2 \mathrm{~g}$ with Figure 2b).

CCK-OPE evokes sequential fusion of $Z G$ s at the apical pole of pancreatic acinar cells. To monitor exocytic events in the acini, we set out to establish a visual assay using FM1-43. A large acinus (more than ten cells) was first tested since it should most reliably preserve the polarized and intercellular morphology necessary to mimic the secretory kinetics occurring in vivo. To image the fluorescent FM1-43 exocytic events occurring in these
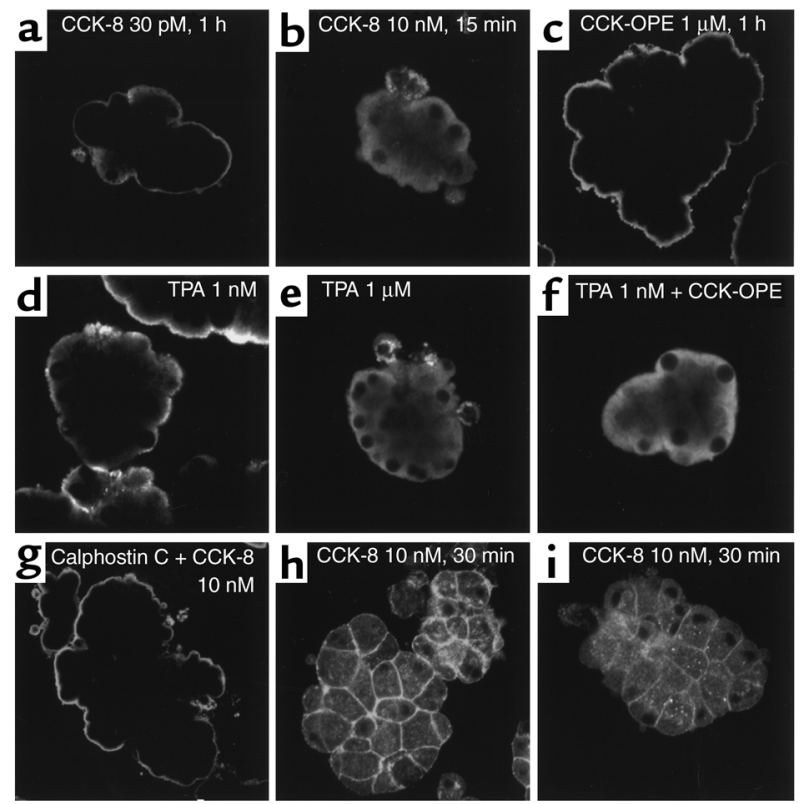

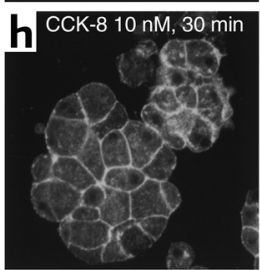

Syntaxin-4

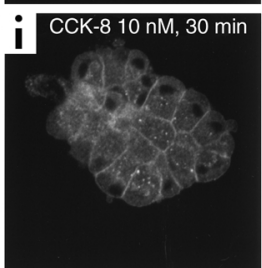

SNAP-23

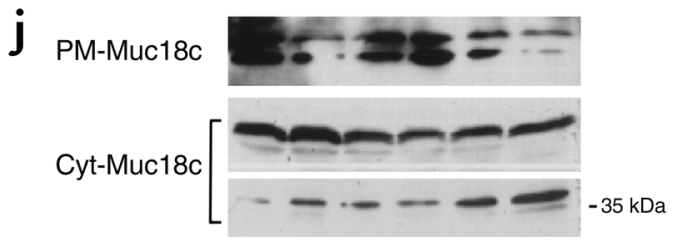

PM-Syntaxin-4

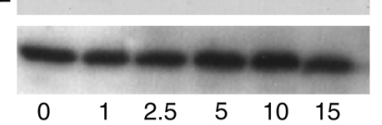

Figure 2

Effects of secretagogues on the cellular localization of Munc18c in dispersed rat pancreatic acini. The dispersed acini were stimulated by the following protocols and were then all labeled with antiMunc18c antibody except for $\mathbf{h}$ and $\mathbf{i}$, which were labeled with anti-syntaxin-4 and anti-SNAP-23 antibodies as indicated in the figure. The cellular locations of these proteins were then visualized by confocal microscopy as described in Methods. (a) Submaximal CCK-8 (30 pM, 1 hour); (b, h, and $\mathbf{i})$ supramaximal CCK-8 (10 nM, 15-30 minutes); (c) supramaximal CCK-OPE ( $1 \mu \mathrm{M}, 1$ hour); (d) 1 nM TPA (30 minutes); (e) $1 \mu \mathrm{M}$ TPA (30 minutes); (f) $1 \mathrm{nM}$ TPA (15 minutes) $+1 \mu \mathrm{M}$ CCK-OPE (30 minutes); (g) calphostin C (500 nM, 40 minutes $)+10 \mathrm{nM} \mathrm{CCK}-8$ (30 minutes). (j) Acini were stimulated with $10 \mathrm{nM} \mathrm{CCK}-8$ at the indicated times and then fractionated into plasma membrane (PM) and cytosol (Cyt) fractions (see Methods). Thirty micrograms protein of the plasma membrane fractions and $40 \mu \mathrm{g}$ of the cytosol fractions were separated on SDS-PAGE and immunoblotted with anti-Munc18c and -syntaxin- 4 antibodies. This is a representative of three independent experiments. 

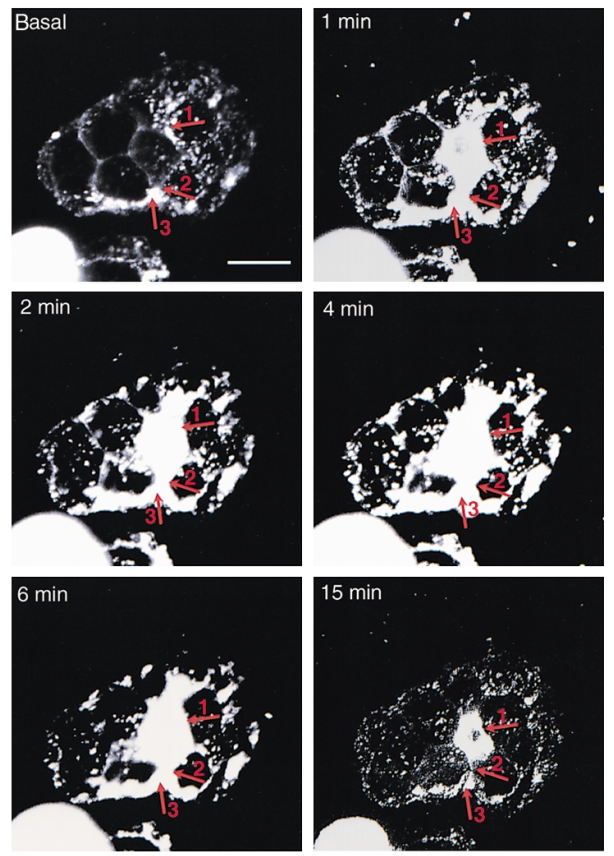

Figure 3

Confocal microscopy of a large acinus showing a 15-minute time course of stimulation by $1 \mu \mathrm{M}$ CCK-OPE. This is a projection of a series of Z-sections across the equatorial plane, which were digitally collapsed using Adobe Photoshop software (Adobe Systems Inc., San Jose, California, USA) to capture the vesicles of adjoining confocal planes. Arrow 1 indicates the acinar ductal lumen; arrow 2 points to its "neck" as it exits out of the acinus, which is marked by arrow 3 . Note that the ductal zymogen protein contents are stained with FM1-43, which fills the ductal lumen. Here, $1 \mu \mathrm{M}$ CCK-OPE stimulation caused a time-dependent (1-6 minutes) increase in fluorescence within the ductal lumen and an increase in the diameter of the lumen. Note tubular structures branching off from the ductal lumen that seem to extend into the apical surfaces of the acinar cells. At 6 minutes, the ductal lumen decreased in size, FM1-43 fluorescence intensity (compared with 4 minutes) decreased, and some of the tubular structures shrank or collapsed. At 15 minutes, the duct was "reduced" in size to resting levels. Note small FM1-43 fluorescent puncta inside the acinar cells at 15 minutes. Bar $=20 \mu \mathrm{m}$.

large acinar units, we used laser confocal imaging to obtain 1- $\mu \mathrm{m}$-thick optical sections. Acini were exposed to $1 \mu \mathrm{M}$ CCK-OPE following a 15 -minute incubation in $4 \mu \mathrm{M}$ FM1-43. We monitored the time course of FM1-43 staining as a projection of all slices to capture the entire exocytic fusion process over 15 minutes. As can clearly be seen in Figure 3, a dramatic increase in FM1-43 fluorescence can be seen within 1 minute in the center of the acinus, consistent with the sites of exocytosis into the lumenal duct. Fluorescence remained high for several minutes but began to weaken after 6 minutes and was significantly reduced within 15 minutes. The extended time of staining may reflect prolonged increases in membrane concentration on the apical surface or direct staining of granular contents, which may require time for dispersal $(27,28)$.

Since repeated exposure to the high-intensity laser is phototoxic to cells, particularly during the time required to capture full thickness Z-sections, we used epifluorescent microscopy imaging, which permits frequent image acquisition required for accurate tracking of real-time agonist-evoked exocytic events occurring at different regions of the acinar cells. Smaller acinar units such as doublets and triplets are suitable for this approach, since they are more likely to be on the same imaging plane. Nonetheless, to demonstrate their utility as acinar models, we obtained a three-dimensional (3D) image of finer $0.6-\mu \mathrm{m}$ confocal cuts of the full thickness of an FM1-43 triplet acinus that was stimulated with $1 \mu \mathrm{M}$ CCK-OPE for 5 minutes (Figure 4a), the time of peak fluorescence observed with the larger acinus in Figure 3. Compared with the phase contrast image, the FM1-43 not only stained the ZGs abutting the apical lumen but diffused contiguously into the deepest and innermost layers of ZGs of each cell. Phototoxic injury, observed as mild blebbing, indeed ensued shortly after this more extensive laser exposure (not shown). Polarity in these acini clusters can be noted by the accumulation of ZGs and actin at the end of the cell (the ZG pole) facing the other cells, which likely represents the ductal remnant (not shown).

As shown in Figure 4, b-d, acquisition of fluorescent images at a frequency of one per second was possible, allowing the data to be analyzed in a similar fashion to that reported for the lactotroph $(27,28)$. Since the epifluorescence images reflect all planes of the acinar cluster, these data are analogous to those obtained with the 3D-reconstructed confocal study (Figure 4a) and the projection of the confocal slices of a larger acinus in Figure 3. At basal stimulation in Figure 4b, FM1-43 lightly stained a small portion of the ZG pole. This may represent constitutive exocytosis, which is known to occur almost entirely at the apical plasma membrane $(33,34)$. Note that this faint staining does not occupy the entire ZG pole in the basal state. In control epifluorescence and confocal microscopy studies with unstimulated acini loaded with FM1-43 and followed for more than 30 minutes at $37^{\circ} \mathrm{C}$, we did not observe further appreciable increases in FM1-43 fluorescence in any region of the acinar cells (data now shown). Stimulation with 1 $\mu \mathrm{M}$ CCK-OPE for 1 minute caused a massive increase in FM1-43 fluorescence that filled the entire ZG pole of all three acinar cells, as was similarly observed in the 3D acinar study shown in Figure 4a.

Next, the changes in fluorescence intensity in the apex, the ZG pole of the three cells, and the entire basal membrane region were tracked and analyzed (Figure 4c). After a short lag time, probably reflecting equilibration of the added agonist to the media, the apical FM1-43 intensity shows a rapid increase (about 12 seconds) to a sustained peak. At the peak plateau level, FM1-43 intensity was about eightfold greater than unstimulated levels. The ZG pole FM1-43 intensity also increased, but in a more gradual manner over a period of about 30 seconds, to a sustained peak that was about fivefold greater than unstimulated levels and that eventually occupied the entire ZG pole of each cell. The basal plasma membrane 
FM1-43 fluorescence intensity remained unchanged initially and, at a later time ( $>1$ minute), only slightly increased to a plateau (Figure 4c). Summation of data obtained from 16 cells ranging from doublets to fourcell acinar clusters showed that the average peak increase in FM1-43 intensity in the apex was $9.8 \pm 1.7$-fold, in the ZG pole was $5.9 \pm 0.9-$ fold, and in the basal membrane was only $1.6 \pm 0.5$-fold (Figure $4 d$ ).

During apical exocytosis, granule membranes and granule contents would be accessible to the extracellular FM1-43. In such a case, the internal FM1-43 should still be continuous to the extracellular space and should be sensitive to washout. However, if endocytosis occurs to retrieve added membranes, FM1-43 would be internalized and inaccessible to washout. As shown in Figure 4, e-j, an acinus was stimulated with $1 \mu \mathrm{M}$ CCK-OPE for 5 minutes (as in Figure 4a) to attain maximal FM1-43 fluorescence intensity within the ZG pole of all of the acinar cells. The acinus was then repeatedly washed with FM1-43-free media. These washes, completed over a period of 15 minutes, removed virtually all of the FM1-43 fluorescence, starting from the plasma membrane, followed by the innermost regions of the $Z G$ pole, and finally the very apex. This pattern of FM1-43 washout could indicate either that all of the granules had collapsed into the apical membrane, or that ZGs fused with the plasma membrane and each other to form stable networks of tubules consistent with those previously observed in ultrastructural studies in the pancreatic acinar cell $(4,35)$. When the fluorescent gain intensity was turned up after the fourth washout (data not shown), we found sparse granular fluorescent spots that could not be washed off. This

\section{Figure 4}

Fluorescence microscopy of $1 \mu \mathrm{M}$ CCK-OPE-stimulated apical exocytosis. (a) A 3D-reconstructed image of $0.6-\mu \mathrm{m}$ confocal cuts of the full thickness of a FM1-43-labeled triplet acinus stimulated by $1 \mu \mathrm{M}$ CCK-OPE for 5 minutes (right). The phase contrast image is also shown (left). (b) Epifluorescence FM1-43 staining of another triplet acinus similarly stimulated with $1 \mu \mathrm{M}$ CCK-OPE. Shown are the phase contrast image (left), basal fluorescent levels (middle), and the fluorescent image after 1 minute of stimulation (right). (c) The same acinus as in $\mathbf{b}$ where images were acquired at 1 frame per second. Regions of interest were drawn as indicated and graphed to show the kinetics of fluorescent changes in real time at these regions - the ZG pole, the apex, and a representative basal plasma membrane region. (d) A graphical analysis of these fluorescent changes (16 cells from doublets to four-cell acini from four experiments) obtained at peak levels. (e-j) The washout of FM1-43 fluorescence from an acinus stimulated with $1 \mu \mathrm{M}$ CCK-OPE to show that the fusion pores of ZGs that have undergone exocytotic fusion remained open. (e) A phase contrast of the acinus. (f) Epifluorescent microscopy of FM1-43 fluorescence of this acinus stimulated by 1 $\mu \mathrm{M}$ CCK-OPE for 5 minutes. This acinus was then subjected to four washes $(\mathbf{g}-\mathbf{j})$ with FM1-43-free media. Note that with each wash, the FM1-43 fluorescence progressively diminished from the innermost portions of the ZG poles of the acinus toward the apical lumen (first [g] to third [i] washes), and finally the apical lumenal fluorescence was washed off (fourth wash $[\mathbf{j}]$ ). Bar $=20 \mu \mathrm{m}$. indicates that only a small amount of FM1-43 had been endocytosed within the 5-minute stimulation.

We used two additional approaches to prove that the changes in apical FM1-43 fluorescence by CCK-OPE stimulation reflect the exocytosis of pancreatic enzymes. First, lowering the temperature to $18^{\circ} \mathrm{C}$, which is known to significantly reduce membrane fusion events, resulted in a complete inhibition of $1 \mu \mathrm{M}$ CCK-OPE-evoked amylase release (data not shown). Also, there was no increase in FM1-43 fluorescence in the acini exposed to $1 \mu \mathrm{M}$ CCK-OPE for at least $20 \mathrm{~min}$ utes at $18^{\circ} \mathrm{C}$ (data not shown). Here, we noted that the slight fluorescence at the apical pole that we normally see in unstimulated acini with FM1-43 alone (Figure 4b) was also inhibited. We interpreted the latter as a decrease in constitutive exocytosis. Second, pretreatment of acini with the $\mathrm{Ca}^{2+}$ chelator BAPTA-AM (10 $\mu \mathrm{M})$ at $37^{\circ} \mathrm{C}$ for 15 minutes, which blocked CCKOPE-evoked cytosolic $\mathrm{Ca}^{2+}$ rise and amylase release, also completely blocked $1 \mu \mathrm{M}$ CCK-OPE-evoked changes in FM1-43 fluorescence in the acini (data not shown).

Supramaximal CCK evokes ectopic exocytosis at the basal plasma membrane. In contrast to CCK-OPE, exposure to supramaximal CCK-8 (10 nM) evoked completely distinct exocytic events. In Figure 5a, we show a confocal image (single section) of a four-cell acinus at basal levels

$\mathbf{a}$

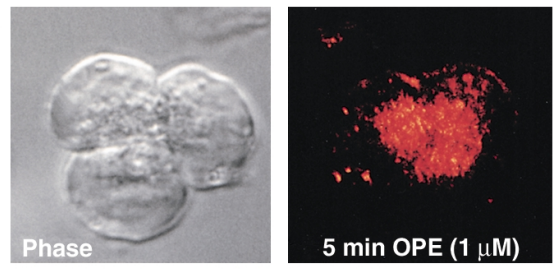

b

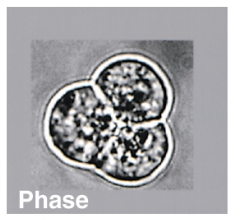

c

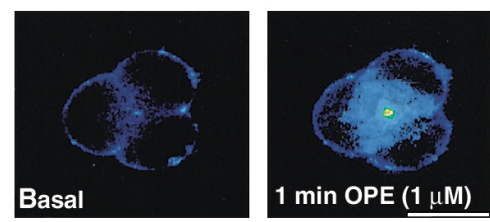

d
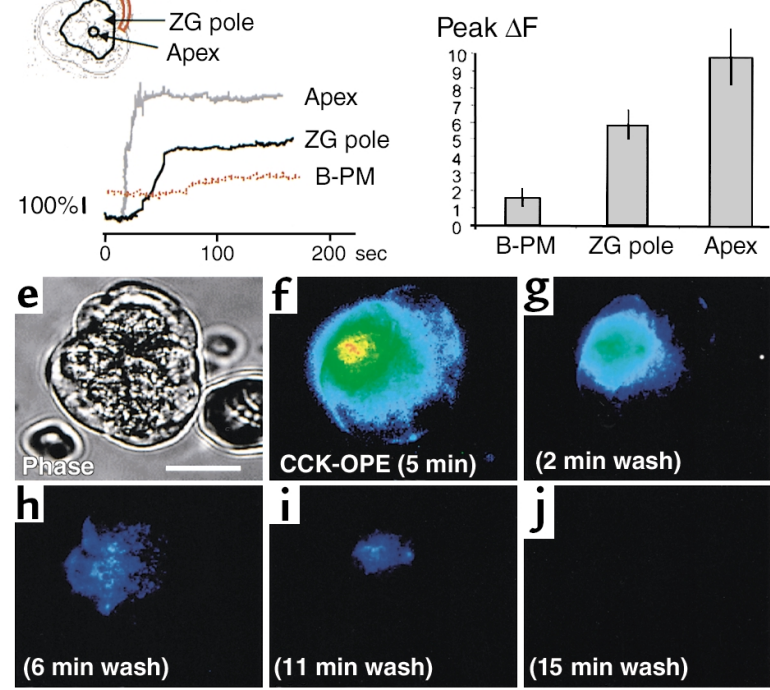
a
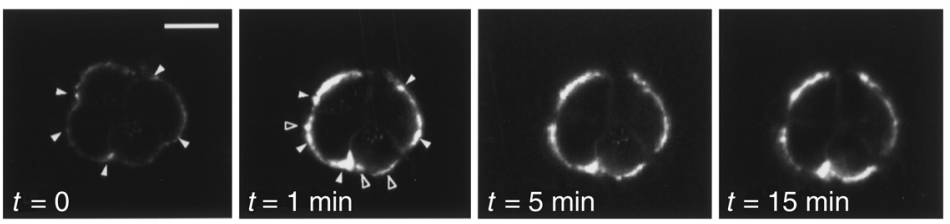

b
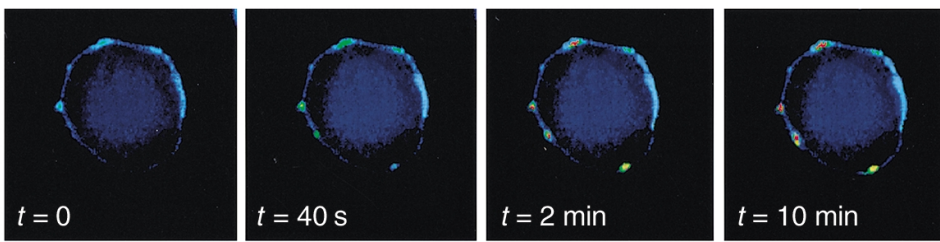

C
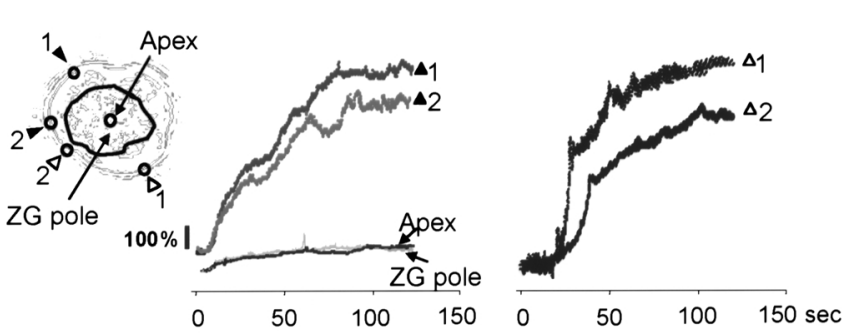

\section{d}

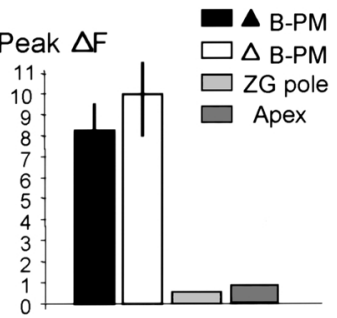

e

$$
\begin{gathered}
\text { Peak } \\
10 \\
9 \\
8 \\
7 \\
6 \\
5 \\
4 \\
3 \\
2 \\
1 \\
0
\end{gathered}
$$
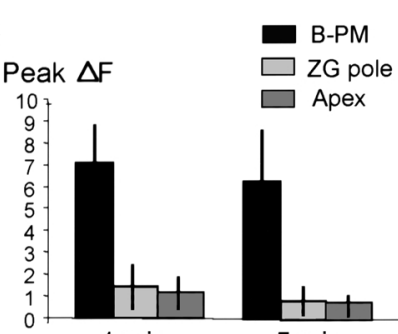

\section{政}

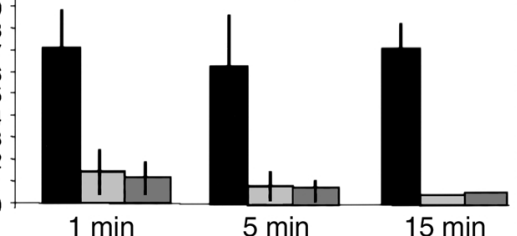

Figure 5

Supramaximal CCK (10 nM) stimulation redirects exocytosis from the apical to the basal plasma membrane. (a) A confocal section across the equatorial plane of a four-cell acinus stained with FM1-43, which was stimulated by $10 \mathrm{nM} \mathrm{CCK}-8$ for 1,5 , and 15 minutes compared with the basal state. Note that most of the membrane hotspots were already present in the cells prior to stimulation (indicated by filled arrowheads) and then increased in intensity and size after stimulation. De novo hotspots not previously present also appeared with CCK stimulation (open arrowheads in the 1-minute image). Bar $=20 \mu \mathrm{m}$. (b and c) Epifluorescence microscopy of a small tripletcell pancreatic acinus stimulated by $10 \mathrm{nM}$ CCK-8. Real-time changes in fluorescence were obtained at $1 \mathrm{frame} / \mathrm{sec}$. (b) The fluorescence images at 0 time, 40 seconds, 2 minutes, and 10 minutes of stimulation. (c) Regions of interest were drawn as indicated on this diagram of the acinus shown in $\mathbf{b}$. The left fluorescence tracing shows two representative hotspots that were present at basal state (filled arrowheads). The right fluorescence tracing shows two de novo hotspots (open arrowheads, see 40-second image in b). (d and e) Graphical summaries of the peak fluorescence data from (d) epifluorescence studies (12 cells, four experiments as in c), including the two populations of basal plasma membrane (B-PM) hotspots ( $n=26$ each, indicated by filled and open arrowheads), and (e) confocal studies (13 cells, three experiments as in a) of $10 \mathrm{nM} \mathrm{CCK-8-evoked} \mathrm{basal} \mathrm{mem-}$ brane FM1-43 exocytosis ( $n=18$ hotspots) at 1,5 , and 15 minutes of stimulation.

and after 1,5 , and 15 minutes of $10 \mathrm{nM} \mathrm{CCK-8}$ stimulation. There was no change in FM1-43 fluorescence in the central apical lumen or the apical poles of the four acinar cells. In contrast, there was a massive increase in the FM1-43 fluorescence of the basal plasma membrane, but only at certain membrane regions and not at all at other membrane regions. Interestingly, when this image of 10 nM CCK-8-stimulated acinus was compared to the unstimulated state, we noted that the most intensely fluorescent regions on the plasma membrane actually occurred at hotspots that were already present before CCK stimulation (indicated in Figure 5 a by filled arrowheads) but that were much fainter in intensity. De novo FM1-43 hotspots (indicated by open arrowheads at 1 minute) also appeared to be recruited and also increased in intensity. In the CCK-OPE-stimulated acini in Figures 3 and 4, the plasma membrane staining with FM143 was also not always uniform and was frequently punctuated by similar faint hotspots, but these membrane hotspots did not appreciably increase in fluorescence intensity with CCK-OPE stimulation. These may reflect sites of constitutive basolateral exocytosis that do not increase in response to CCK-OPE. Supramaximal CCK appears to redirect apical exocytosis to defined sites on the basal membrane. It therefore appears that basal membrane exocytosis does not occur at random sites on the plasma membrane.
In Figure 5, b and c, we performed epifluorescence imaging to monitor the kinetics of basal membrane exocytosis. Stimulation with $10 \mathrm{nM}$ CCK- 8 caused the basal membrane hotspots to abruptly increase in intensity in a time-dependent manner, monitored for up to $10 \mathrm{~min}$ utes of stimulation (Figure 5b). In Figure 5c, at the two representative basal plasma membrane hotspots previously present at the basal state (compare diagram with $t=0$ image in Figure 5b), the FM1-43 fluorescence (see left tracing indicated by filled arrowheads in Figure 5c) was analyzed and observed to increase in series of twoto fourfold steps over the base-line levels. These fluorescence intensity changes between these two basal membrane hotspots were remarkably synchronous. The two representative de novo membrane hotspots (compare diagram with $t=40 \mathrm{~s}$ image in Figure $5 \mathrm{~b}$ ), which appeared after 20 seconds of stimulation (see right tracing indicated by open arrowheads in Figure 5c) also increased in intensity in a very similar manner, with four- to fivefold step increases, although the second step rise seemed slower. The peak fluorescence intensities between these two populations of hotspots (Figure 5d) were similar $-8.4 \pm 1.3$-fold and $10.0 \pm 2.1$-fold, respechotspots exhibited these patterns. Like the lactotroph studies, these hotspots also appeared as solid spots, not rings, suggesting that the FM1-43 was staining both the tively. The vast majority of the basal membrane 
ZG membranes and the granule cores, as was also reported in the lactotrophs $(27,28)$. This increase in fluorescence eventually plateaued within 2 minutes with no further increase even with continued CCK stimulation of up to 10 minutes (see Figure $5 \mathrm{~b}$ ). In contrast to $1 \mu \mathrm{M}$ CCK-OPE, there was little change in the FM1-43 fluorescence in the apex or the ZG poles of this acinus (left tracing in Figure 5c and graphical summary in Figure 5d), which would indicate that supramaximal CCK stimulation caused a blockade in apical exocytosis. This is consistent with previously reported ultrastructural studies (4). Figure 5e shows a graphical summary of confocal studies of all basal membrane hotspots analyzed by confocal imaging ( 18 hotspots from 13 cells from 3 acinar clusters) at 1,5 , and 15 minutes of stimulation (as in Figure 5a). These studies demonstrated an average sevenfold increase in intensity with little fluctuation over the course of 15 minutes, whereas the apical membrane and ZG pole fluorescence remained unchanged or even seemed slightly decreased during the 15 minutes of $10 \mathrm{nM}$ CCK-8 stimulation. To demonstrate that basal membrane exocytosis is also a $\mathrm{Ca}^{2+}$ - and temperature-dependent event, as was the case with apical exocytosis, we showed that a decrease of the temperature to $18^{\circ} \mathrm{C}$ or $\mathrm{Ca}^{2+}$ chelation with $10 \mu \mathrm{M}$ BAPTA-AM completely blocked these $10 \mathrm{nM}$ CCK-evoked exocytic FM1-43 fluorescence events, and blocked amylase release as well (data not shown).

We next examined the effects of TPA plus CCK-OPE stimulation on the pattern of FM1-43 fluorescence changes. In Figure 6a, we show a confocal section of a four-cell acinus that was preincubated with $1 \mathrm{nM}$ TPA (15 minutes, $37^{\circ} \mathrm{C}$ ) and that showed no change in FM1-43 fluorescence compared with before TPA treatment (not shown). When $1 \mu \mathrm{M}$ CCK-OPE was added, fluorescence

\footnotetext{
Figure 6

Supramaximal CCK-evoked basal membrane exocytosis by a PKCmediated pathway. (a) A confocal section across the equatorial place of a four-cell acinus stained with FM1-43, which was preincubated with $1 \mathrm{nM}$ TPA for 15 minutes $37^{\circ} \mathrm{C}$ and then combined with $1 \mu \mathrm{M}$ CCK-OPE. Images were taken during the $1 \mathrm{nM}$ TPA preincubation, and the fluorescence was identical to basal levels before exposure to TPA (not shown). Many of the basal membrane hotspots were already present prior to CCK-OPE stimulation and then increased in intensity and size after stimulation (filled arrowheads); only a few more de novo membrane hotspots appeared later (open arrowheads). Bar $=20 \mu \mathrm{m}$. (b) Epifluorescence microscopy (1 frame per second) performed on a doublet-cell acinus subjected to the same protocol of preincubation with $1 \mathrm{nM}$ TPA, followed by addition of $1 \mu \mathrm{M}$ CCK-OPE. A phase contrast image of the acinus and the regions of interest drawn on this acinus for analysis are shown. 0 time is when $1 \mu \mathrm{M} \mathrm{CCK-OPE} \mathrm{was} \mathrm{added}$ into the $1 \mathrm{nM}$ TPA-containing media. The 15-minute TPA preincubation is not shown. Note the synchronous increase in the fluorescence intensity of the basal plasma membrane hotspots (B-PM1 and B-PM2); in contrast, there was no change in the FM1-43 fluorescence intensity in the apex or ZG poles of the acinus. (c) A graphical summary of the peak fluorescence data obtained from several confocal experiments performed as in a $(n=15$ basal membrane hotspots from 9 cells; three separate experiments).
}

hotspots appeared at the basal plasma membrane (indicated by arrowheads) and not in the apical lumen or ZG pole as previously observed in Figure 4. This pattern of basal membrane fluorescence was exactly the same to that observed with supramaximal CCK stimulation (Figure 5). Also, as with supramaximal CCK stimulation, while many of these basal membrane hotspots occurred at sites that were already present in resting cells (indicated by filled arrowheads, Figure 6a, left and right images), additional de novo membrane hotspots were also recruited that were not previously present (indicated by open arrowheads, Figure 6a, right image). In Figure 6b, we show an epifluorescence analysis of a doublet cell subjected to this stimulatory protocol to reveal the kinetics of exocytosis. Two of the basal membrane hotspots (as indicated) showed four- and sixfold increases in intensity. The increase in FM1-43 fluorescence intensity of these hotspots was also in a stepwise (note shouldering at halfway up) and synchronous fashion. A graphical summary $(n=15$ hotspots from 9 cells in Figure 6c) showed a mean sixfold increase in peak intensity of these basal membrane hotspots. Of note, there were no changes in the apical fluorescence (central apical lumen and apical ZG poles) with this stimulation protocol, as was the case with supramaximal CCK stimulation (Figure 5).

To unequivocally demonstrate that the basal plasma membrane FM1-43 fluorescence hotspots are indeed ZG exocytic events, we performed double labeling of amylase with FM1-43 (Figure 7) in nonpermeabilized acini stimulated with supramaximal CCK-8 $(100 \mathrm{nM})$ concentrations. This experiment was performed without mem-
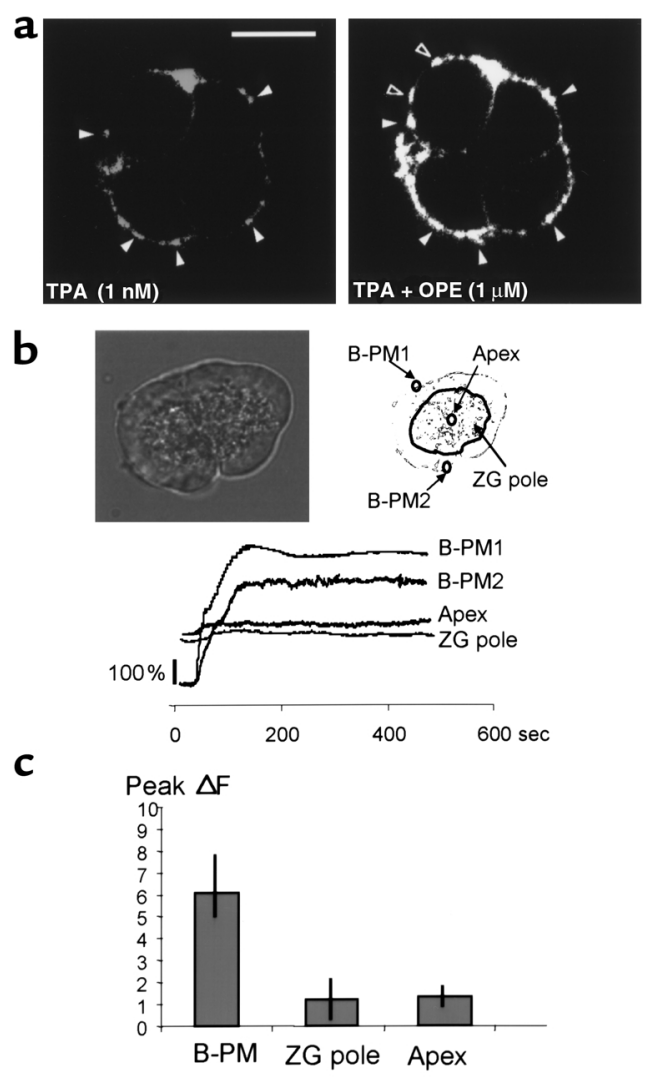

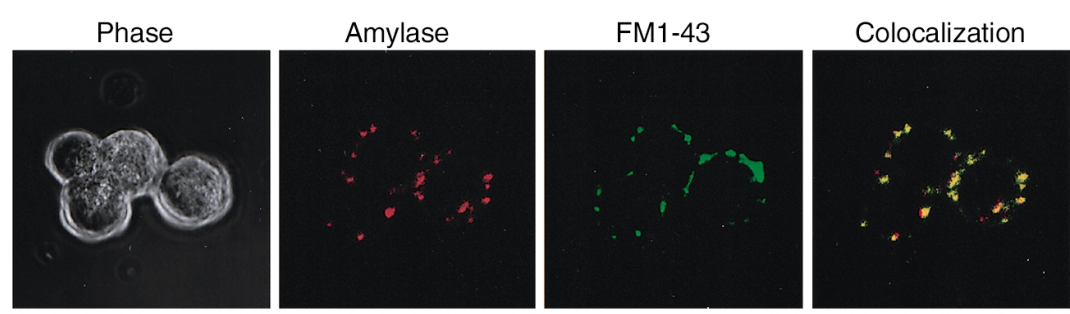

Figure 7

Supramaximal CCK (100 nM)-evoked basal membrane FM1-43 hotspots persist and immunostain precisely with exocytosed amylase at the basal plasma membrane. The acini were stimulated with $100 \mathrm{nM} \mathrm{CCK}$ for 20 minutes $\left(37^{\circ} \mathrm{C}\right)$, followed by costaining with antiamylase antibody (red) and FM1-43 (green) as described in Methods. The phase contrast image of the acinar cells is shown to demonstrate their health after the CCK stimulation. Superimposition of the anti-amylase and FM1-43 shows a precise colocalization (yellow spots) of most of the basal membrane FM1-43 hotspots and amylase. Bar $=20 \mu \mathrm{m}$.

brane permeabilization so that the anti-amylase antibody would only label the exocytosed ZGs and not the intracellular amylase; furthermore, this would avoid FM1-43 labeling of internal membranes. Primary (antiinsulin) and secondary (FITC-labeled) antibody controls were negative at unstimulated and $10 \mathrm{nM}$ CCK-8-stimulated conditions (5 minutes) (data not shown). Here, the basal membrane FM1-43 (green) and amylase (red) staining almost completely colocalized (indicated by yellow spots; Figure 7). No staining of FM1-43 or of amylase was noted in the apical ZG poles of the acinar cells. In vivo pancreatitis caused by supramaximal CCK-8 disrupts Munc18c plasma membrane localization. We next determined whether supramaximal cerulein could cause changes in Munc18c in intact rat pancreatic tissue similar to those shown in Figure 2. Perfusion with saline (or submaximal secretory concentrations of cerulein; data not shown) did not affect Munc18c localization at the basolateral plasma membrane (Figure 8a) or the location of actin (Figure 8b). Note that the Munc18c staining in the lateral plasma membranes between acinar cells of the whole tissue is more consistently present than in the dispersed acini (Figures 1 and 2). F-actin is abundant in the apical plasma membrane of the acinar cells in intact rat pancreas and shows a weak but intact signal in the basolateral plasma membrane (Figure $8 \mathrm{~b}$ ). Infusion of supramaximal cerulein caused a time-dependent progressive displacement of Munc18c from the plasma membrane of the acinar cells. As early as 10 minutes of infusion (Figure $8 \mathrm{~d}$ ), we detected a generalized displacement of Munc18c from the plasma membrane into the cytosol. At 1 hour of supramaximal cerulein infusion (Figure 8g), Munc18c displacement, observed as greatly diminished plasma membrane levels of Munc18c, was observed; Munc18c displaced from the plasma membrane maximal at 2 hours of infusion (Figure 8j). The apparent absence of a detectable Munc18c signal in the cytosol in Figure 8, $g$ and $j$, may be due to a combination of cytosolic degradation (as shown in Figure 2j) and dilution.

The progression of Munc18c displacement from the plasma membrane correlated very well with the progres- sion of actin disassembly (Figure 8, e, $\mathrm{h}$, and $\mathrm{k}$ ), which is well known to occur in this pancreatitis model (30, 31). Also, parallel histologic sections obtained from these pancreata demonstrate progressive changes of edematous pancreatitis. Only sparse cytoplasmic vacuoles (arrows) were seen at 10 minutes of cerulein infusion (Figure 8f), and these vacuoles became progressively larger (arrows) at 1 hour of cerulein infusion (Figure 8i) and generalized at 2 hours cerulein infusion (Figure 8, part 1). Of note, after only 10 minutes of cerulein infusion, when mild membrane dissociation of Munc18c is observed in most acinar cells (Figure 8d), only minimal actin disassembly (Figure 8e) and few vacuolations (Figure $8 \mathrm{f}$ ) were seen. This would suggest that Munc18c displacement from the membrane occurs earlier and/or is more generalized than these morphologic features of acute pancreatitis. These in vivo effects on Munc18c behavior in the pancreas following supramaximal cerulein infusions parallel those in vitro in the dispersed acini model above. However, maximal effects were reached later ( $\sim 2$ hours) in vivo than in the in vitro model (30 minutes), which may be due to degradation of the hormone by serum proteases or delays in achieving high tissue levels in vivo. In contrast to Munc18c, syntaxin-4 (Figure 8m) and SNAP-23 (Figure 8o) locations at the basolateral plasma membrane were not affected even at 4 hours of supramaximal cerulein infusion, when compared with saline-infused pancreas (not shown). These results are consistent with the confocal (Figure 2, $h$ and i) and subcellular fractionation (Figure 2j) studies performed on dispersed acini. Taken together, these studies suggest that displacement of Munc18c from the acinar plasma membrane by supramaximal cerulein treatment exposes the basolateral membrane syntaxin- 4 to permit binding to plasma membrane SNAP-23 and potentially also to ZG membrane VAMP-2, resulting in basolateral exocytosis (as shown in Figure 5, a-e).

Minimal PKC activation in vivo causes CCK-OPE to induce pancreatitis. Intraperitoneal injection of low concentrations of TPA ( $1 \mathrm{nmol} / \mathrm{kg}$, Figure $9 \mathrm{c})$ or supramaximal CCK-OPE $(10 \mu \mathrm{g} / \mathrm{kg}$, Figure 9e) into the rats had no detectable effects on Munc18c location when compared with control saline infusion (Figure 9a). However, when the injection of low TPA concentrations $(1 \mathrm{nmol} / \mathrm{kg})$ was followed after 10 minutes by the injection of supramaximal CCK-OPE $(10 \mu \mathrm{g} / \mathrm{kg})$, Munc18c displacement from the plasma membrane was observed (Figure 9g). The more common pattern of Munc18c displacement consisted of greatly reduced plasma membrane levels of Munc18c (arrowheads in Figure 9g) similar to those observed with treatment with supramaximal cerulein concentrations (Figure 9i). A somewhat less common pattern was a more normal Munc18c intensity in the 
plasma membrane that, however, appeared to be undergoing either fragmentation or dissociation from the plasma membrane (arrows in Figure 9g), as similarly observed in the dispersed acini experiment (Figure 2f). About $60 \%$ of the pancreatic acini in any given section had these changes. The rest of the acini had intact and normal intensity of Munc18c signal on the plasma membrane. This is in contrast to the generalized involvement caused by supramaximal cerulein treatment ( 2 hours in Figure $8 j$ and 4 hours in Figure 9i).

To determine whether these treatment protocols could cause pancreatitis, we examined the histology of each of these rat pancreatic tissues. Rats intraperitoneally injected with saline (Figure 9b), low TPA (Figure 9d), or supramaximal CCK-OPE (Figure 9f) had normal pancreatic histology. Exposure to supramaximal cerulein (Figure 9j) caused typical changes of mild edematous pancreatitis $(4-6,36,37)$, including interstitial edema and extensive formation of small and large intracytoplasmic vacuoles (arrows) within many of the acinar cells (as in Figure 8, parts i and l). Of note, the pancreatic tissue exposed to low TPA concentrations $(1 \mathrm{nmol} / \mathrm{kg})$ for 10 minutes followed by CCK-OPE injection (10 $\mu \mathrm{g} / \mathrm{kg}$ ) and after 4 hours the pancreas was surgically excised (Figure 9h), which demonstrated similar changes to those seen with the 10-minute supramaximal cerulein infusion (Figure 8f). These changes included a very mild degree of acute pancreatitis with fewer acinar cells containing smaller cytoplasmic vacuoles (arrows, Figure 9h). These infusion protocols did not cause a change in the behavior of basolateral membrane syntaxin-4 and SNAP-23 (data not shown), which appeared the same as that shown in Figure 8, $\mathrm{m}$ and o.

Basolateral exocytosis into the interstitial space is believed to account for the early appearance of pancreatic proteases (e.g., trypsin or carboxypeptidase) or other digestive enzymes (e.g., amylase or lipase) in the circulation during acute pancreatitis, which are used as diagnostic tests for human acute pancreatitis (38).

\section{Figure 8}

Confocal microscopy localization of Munc18c and actin and histology of pancreatic tissues from rats treated with supramaximal cerulein concentrations. Rats were intravenously infused with saline control (a-c) or supramaximal cerulein $(10 \mu \mathrm{g} / \mathrm{kg} / \mathrm{h})$ for 10 minutes $(\mathbf{d}-\mathbf{f}), 1$ hour $(\mathbf{g}-\mathbf{i}), 2$ hours $(\mathbf{j}-\mathbf{I})$, or 4 hours $(\mathbf{m}-\mathbf{o})$. The rats were immediately perfused with a fixative and the pancreata removed and prepared for confocal microscopy (see Methods) and histology (hematoxylin and eosin stain). For the confocal studies, the pancreatic tissue sections were labeled with anti-Munc18c (a, d, g, and $\mathbf{j})$ and doublelabeled with FITC-phalloidin ( $\mathbf{b}, \mathbf{e}, \mathbf{h}$, and $\mathbf{k}$ ). Note the progressive displacement of Munc18c and its diminished levels in the pancreatic acinar plasma membranes. These changes of Munc18c correlate to the progressive actin disassembly and the formation of cytoplasmic vacuoles (arrows in $\mathbf{f}$ and $\mathbf{i}$ ) in the acinar cells. The pancreatic acinar cells nonetheless remain completely intact $(\mathbf{f}, \mathbf{i}$, and $\mathbf{I})$. ( $\mathbf{m}-\mathbf{o})$ Pancreata from rats treated with supramaximal cerulein for 4 hours. Treatment did not affect syntaxin-4 (m) or SNAP-23 (o) plasma membrane locations but did cause actin disassembly $(\mathbf{n})$.
We therefore examined the serum amylase levels in these rats prior to sacrifice. When these rats were subjected to infusion with control saline, low TPA $(1 \mathrm{nmol} / \mathrm{kg})$, and supramaximal CCK-OPE, their serum amylase levels in each case were measured to be uniformly normal, at 40U/1. Supramaximal cerulein infusion elevated serum amylase to $180 \mathrm{U} / \mathrm{l}$, whereas low TPA $(1 \mathrm{nmol} / \mathrm{kg})+$ supramaximal CCK-OPE $(10$ $\mu \mathrm{g} / \mathrm{kg}$ ) elevated serum amylase to $100 \mathrm{U} / \mathrm{l}$, both of which are within the pancreatitis range. The lower amylase level caused by the low TPA + CCK-OPE treatment would at least in part be accounted for by the less generalized ( $60 \%)$ and less pronounced Munc18c displacement from the acinar plasma membrane (Figure $9 \mathrm{~g}$ ) and the milder histological changes of pancreatitis (Figure 9h). In contrast, the higher amylase levels caused by supramaximal cerulein infusion correlated well with the more generalized Munc18c displacement (Figures $9 \mathrm{i}$ and $8 \mathrm{j}$ ) and advanced histological changes of mild pancreatitis (Figure 9j). These studies therefore demonstrate a more generalized and extensive
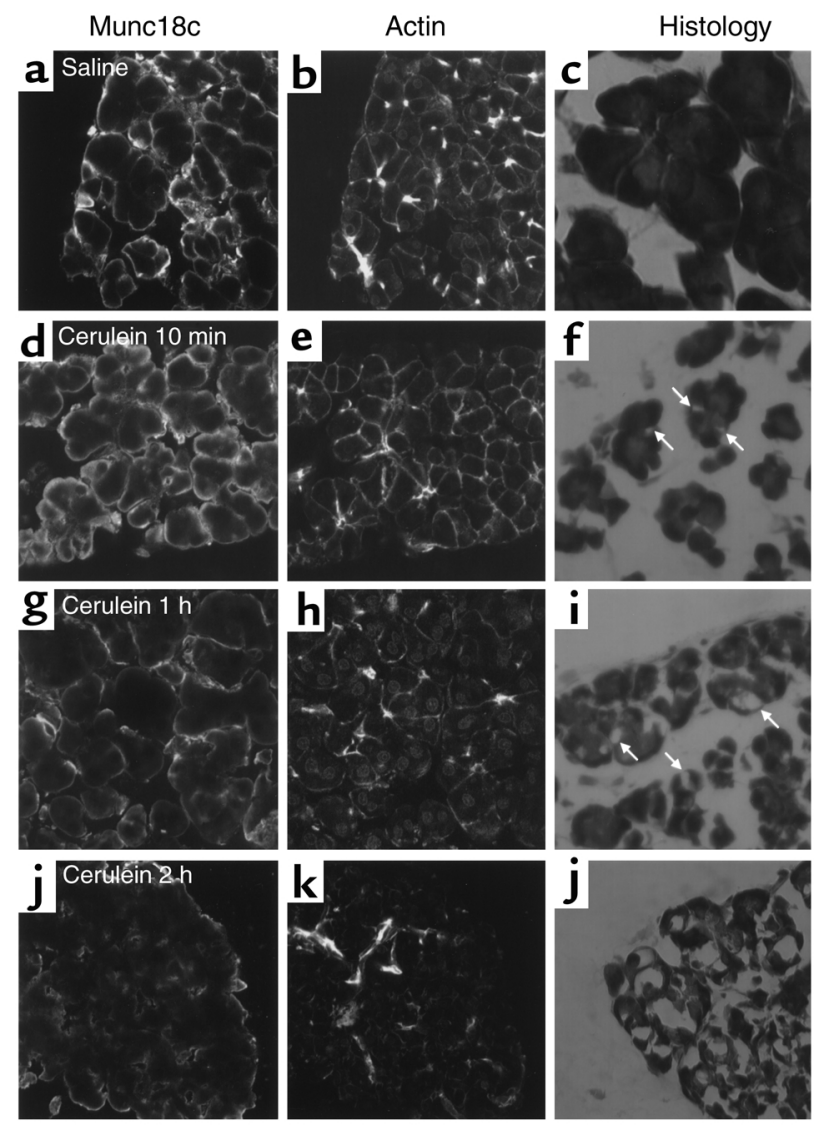

Syntaxin-4

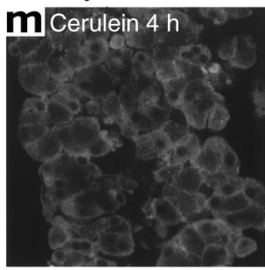

Actin

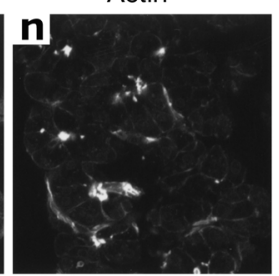

SNAP-23

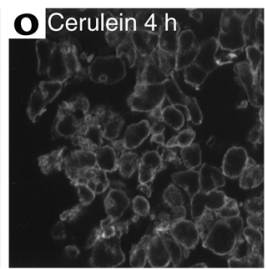



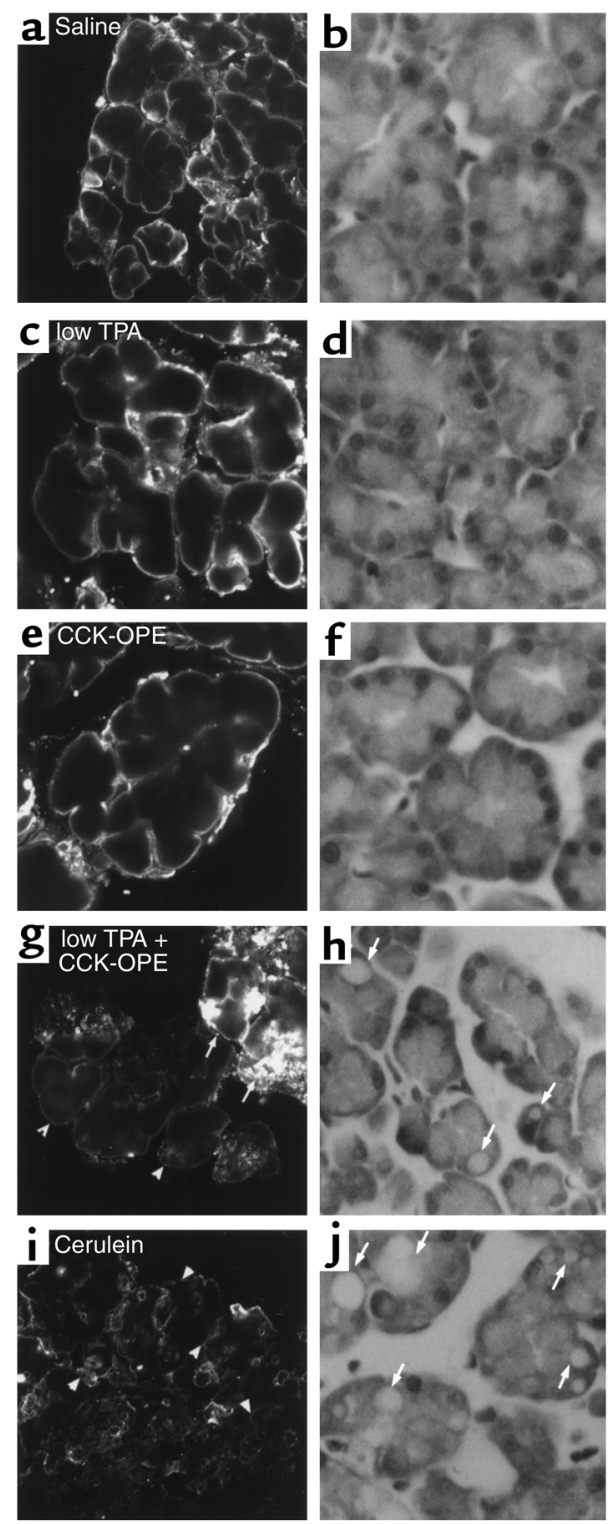

Munc18c dissociation from the plasma membrane than the cytoplasmic vacuole formation in the mild edematous pancreatitis caused by either supramaximal cerulein or low TPA + CCK-OPE.

\section{Discussion}

The minimal machinery for membrane fusion consists of a cognate set of $\mathrm{v}$ - and $\mathrm{t}$-SNAREs on opposing membranes capable of forming a stable complex, and the formation of this complex is thought to provide the energy to cause membrane fusion $(12,13)$. The best-characterized SNARE complex, composed of syntaxin-1a, VAMP-2, and SNAP-25, drives exocytosis of synaptic vesicles in the neuron $(12,13)$. In the pancreatic acinar cell, we $(18-21,39)$ and others $(40)$ have previously reported the presence and cellular locations of distinct sets of $\mathrm{v}$ - and t-SNAREs to apical exocytosis, homotypic ZG fusion, and basolateral membrane exocytosis (15). Specifically, t-SNAREs

\section{Figure 9}

Minimal PKC activation in vivo causes CCK-OPE to mimic cerulein in inducing pancreatitis in rats. Rats were intraperitoneally injected with 50-100 $\mu \mathrm{l}$ of saline control ( $\mathbf{a}$ and $\mathbf{b})$, low TPA ( $1 \mathrm{nmol} / \mathrm{kg}$ ) (c and $\mathbf{d})$, supramaximal CCK-OPE $(10 \mu \mathrm{g} / \mathrm{kg})(\mathbf{e}$ and $\mathbf{f})$, low TPA $(1 \mathrm{nmol} / \mathrm{kg})+$ CCK-OPE $(10 \mu \mathrm{g} / \mathrm{kg})$ ( $\mathbf{g}$ and $\mathbf{h}$ ), or supramaximal cerulein $(10 \mu \mathrm{g} / \mathrm{kg}$ ) concentrations (i and $\mathbf{j})$. Four hours after the injections of these reagents, the rats were treated and the pancreata removed as in Figure 8 (and in Methods). A portion of each pancreas was prepared for confocal microscopy studies $(\mathbf{a}, \mathbf{c}, \mathbf{e}, \mathbf{g}$, and $\mathbf{i})$ to determine Munc18c localization, and another portion was prepared for histologic studies ( $\mathbf{b}, \mathbf{d}, \mathbf{f}, \mathbf{h}$, and $\mathbf{j}$ ) (hematoxylin and eosin staining) to determine the early morphologic features of mild edematous pancreatitis. In the low TPA + CCK-OPE protocol, the rat was first injected with $1 \mathrm{nmol} / \mathrm{kg}$ TPA and, after 10 minutes, was injected with CCK-OPE. Note the similar changes in the TPA + CCK-OPE-treated ( $\mathbf{g}$ and $\mathbf{h}$ ) and the cerulein-treated (i and $\mathbf{j}$ ) pancreatic tissues, including Munc18c disruption (arrows) and diminished plasma membrane levels (arrowheads) in $\mathbf{g}$ and $\mathbf{i}$, and the formation of small and large cytoplasmic vacuoles (arrows) in $\mathbf{h}$ and $\mathbf{j}$. These changes caused by supramaximal cerulein ( $\mathbf{i}$ and $\mathbf{j}$ ) were, however, more marked and generalized than the changes in the low TPA + CCK-OPE-treated tissues ( $\mathbf{g}$ and $\mathbf{h}$ ).

syntaxin-2, -3 , and -4 have been localized to the apical membrane, ZG, and basolateral membrane, respectively (21), and their role in mediating these membrane fusion events was demonstrated with an elegant in vitro membrane fusion assay (40). The t-SNARE SNAP-23 is present in the acinar plasma membrane and $Z G$ membrane $(20,21)$ and is involved in CCK-OPE-evoked amylase secretion (39). Stimulation of mast cells caused the relocation of SNAP-23 from the plasma membrane to the secretory granules via lamellipodia, which was required to link granule-plasma membrane and granule-granule fusions in the process of compound exocytosis (41). The only v-SNARE present on the ZG membrane shown to be involved in $\mathrm{Ca}^{2+}$-evoked enzyme secretion is VAMP-2 $(18,19)$. In fact, the basal membrane-restricted forms, syntaxin-4 and SNAP-23, have been shown to form SNARE complexes with VAMP-2 in vitro $(21,22)$ and function in mediating GLUT4 translocation in adipocytes (42-44).

Cognate SNARE proteins may not be sufficient to mediate the specificity of distinct membrane fusion events, and additional chaperone proteins are required to assist in the assembly and disassembly of the exocytic SNARE complexes (45). Rab proteins, in particular Rab3, have been postulated to play a role in regulated exocytosis in the pancreatic acinar cell $(34,46,47)$ and can potentially interact with acinar SNARE proteins (18). Jamieson et al. have recently reported that Rab3 is required for actin coating of the apical ZGs (46). Whereas more work is needed to identify additional regulatory proteins involved in apical exocytosis, we have gained further insights into a regulator, Munc18c, that we believed to be involved in basolateral exocytosis. First, it should be noted that basolateral exocytosis rarely occurs in the pancreatic acinar cell (33), despite the presence of the cognate SNAREs syntaxin-4, SNAP-23, and VAMP-2. This suggests that the regulatory protein must also serve to prevent this 
basolateral membrane SNARE complex from assembling spontaneously (15). Munc18 proteins are candidates for such a role (15). Non-neuronal Munc18 isoforms, including Munc18b and Munc18c, have been reported $(23,24)$. In adipocytes, Munc18c is the only Munc18 protein that binds syntaxin- 4 with high affinity, and blocks syntaxin binding to SNAP-23 and VAMP-2 (24-26). In neurons, Munc18a binds with very high affinity to syntaxin-1a and is a very potent inhibitor of SNARE complex assembly (14). It is possible that dissociation of Munc18c from the basal membrane may be due to direct phosphorylation, since the human platelet Sec1 protein (called PSP) was recently reported to have $92 \%$ sequence identity with Munc18c at the peptide level (48) and may be the human homolog of Munc18c. The PSP sequence contains a motif for PKC phosphorylation at residues 128-131 (KSIR), which is conserved at the same residues 128-131 in Munc18c. PSP was shown to be phosphorylated in vitro by $\mathrm{PKC}$ incorporating 0.9 mole $^{\mathrm{PO}_{4}}$ per mole of protein (48). Moreover, this PKC-mediated phosphorylation inhibits PSP from binding syntaxin-4, as this interaction regulated vesicle docking and secretion in platelets. Parallel observations were reported for Munc18a, whereby in vitro PKC phosphorylation of Munc18a inhibited its binding to syntaxin-1a $(16,17)$. Attempts to measure Munc18c phosphorylation in the pancreatic acini have been hampered by the high protease content of this tissue.

Evidence that basal membrane Munc18c may act to shield syntaxin- 4 to prevent basal exocytosis of ZGs comes from our measurements of exocytosis in vitro using FM1-43. This assay was first shown to visualize exocytic events in the neuroendocrine lactrotrophs (27, 28). In the lactotroph, FM1-43 labeled not only secretory granule membranes but also the granule core contents, which explains why the exocytic hotspots appeared as solid spots $(27,28)$. These solid spots are due to staining of some undefined hydrophobic components within the dense core matrix, which Betz and coworkers suggested could include the secreted prolactin $(27,28)$. Indeed, prolactin immunolocalized precisely with the FM1-43 $(27,28)$. In the pancreatic acinar cell, the size of the FM1-43 fluorescent exocytic hotspots (at the acinar basal membrane), including the larger ones that may result from compound exocytosis, and the secretory kinetics were almost identical to those of the lactrotroph. Furthermore, the precise immunolocalization of the amylase to the FM1-43 hotspots suggests that this digestive enzyme might share some undefined physicochemical property with the prolactin in being labeled with FM1-43. These remarkable similarities between the two cell types in the exocytic events visualized by FM1-43 fluorescent imaging clearly indicate that this optical assay is useful in monitoring the distinct exocytic events in the pancreatic acinar cell (29) in real time and with high spatial resolution with the use of epifluorescence and confocal microscopy. Nonetheless, the regulation of exocytosis is very different in the lactotroph and in polarized pancreatic acinar cells.

Regulated apical exocytosis in pancreatic acinar cells is very efficiently effected by physiologic CCK (or CCK$\mathrm{OPE}$ ) concentrations $(3,8)$ despite the limited surface area of the apical plasma membrane. Homotypic fusion mechanisms would be an efficient means to rapidly deliver large quantities of ZG contents into the duct, since the large granules would not need to be moved toward and make contact with the apical plasma membrane but could fuse with adjacent granules. Several lines of evidence have recently been reported to support this notion of sequential apical homotypic ZG fusions and the formation of stable fusion pores. Using atomic force microscopy, Schneider et al. (49) showed that the exocytic pores formed by transient fusion of the ZG membrane with the plasma membrane, rather than complete collapse into the surface membrane and subsequent massive expansion of the lumenal membrane. J. Jamieson's laboratory showed by electron microscopy that carbachol stimulation caused the formation of large vacuolar structures within the acinar cells that were continuous with the lumen (32). Of note, a large amount of amylase accumulated in these vacuolar structures but was not extruded into the acinar ductal lumen in the continued presence of carbachol or in the presence of actin-depolymerizing agent cytochalasin $\mathrm{D}$ (32). The zymogen core contents were extruded only after carbachol or cytochalasin D was withdrawn, which suggests that the expulsion of the core proteins is dependent on an active actinomyosin contractile system. While our manuscript was being prepared, a report using multiphoton excitation imaging methods showed similar findings of the apical exocytic events, including sequential ZG fusion, in intact pancreatic acini (50). In fact, our 3D-reconstructed image of a confocal FM1-43 study of OPE-stimulated acinus (Figure 4a) is identical to that shown by the multiphoton excitation imaging study (50). In both apical and basal secretion events, we observed a series of stepwise increases in fluorescence. This may indicate some unknown pulsatile signaling event, or may reflect the sequential or simultaneous fusion of large numbers of ZGs, as would be the case if they had previously fused by homotypic processes before fusion with the plasma membrane.

Acini stimulated with supramaximal CCK concentrations actually result in an inefficient exocytosis observed as an inhibition of secretion from maximal levels $(3,4)$. Ultrastructural studies had demonstrated that supramaximal CCK stimulation caused an apical blockade with only rare exocytosis events seen at the lateral membrane (4), a controversial observation. The substantial enzyme release observed in cultured acini during supramaximal CCK stimulation ( $70 \%$ of maximal) could therefore not be explained until now (3). Our study demonstrates that supramaximal CCK causes an aberrant targeting of the ZGs to the basal membrane. Of note, although the acinar basal membrane is very large, exocytosis occurred initially at a few fixed domains 
rather than throughout the basal membrane. These restricted exocytic domains may in part explain the difficulty in observing these by ultrastructural studies (4). Low levels of FM1-43 staining were observed in discrete sites on the membranes of unstimulated stimulated cells, but these did not increase in brightness following CCK-OPE stimulation. It remains possible that these are sites of constitutive basal membrane secretion, since previous studies have shown that basolateral secretion can occur at defined sites on the plasma membrane of polarized MDCK cells (51). It will be of interest to determine whether supramaximal stimulation results in subversion of the constitutive pathway by the regulated ZGs.

Dissociation of Munc18c from the plasma membrane by CCK activation of PKC would free syntaxin-4 to bind membrane SNAP-23 and granule VAMP-2 to form the exocytic complex. This is demonstrated by the temporal correlation of supramaximal CCK-induced displacement of Munc18c from the plasma membrane into the cytosol (Figure 2) and basal membrane exocytosis (Figure 5). Although relatively little is known about Munc18c, its function and regulation likely parallel those of its neuronal isoform, Munc18a/nSec1. It is now known that Munc18a does not simply act as a negative regulator that restricts the synaptic vesicle from exocytotic fusion; rather, its dissociation from syntaxin-1A changes syntaxin-1A from a "closed" to an "open" configuration capable of binding its cognate SNARE proteins (52). This would explain the previous studies suggesting that Munc18a possesses apparently conflicting positive and negative roles in exocytosis (14, $53,54)$. Moreover, Munc18a not only plays this complex role in SNARE complex assembly but, as was recently shown, plays a role in the control of fusion pore dynamics as well (55).

Interestingly, an exaggeration of basal membrane exocytosis, presumably resulting from the perturbation of these PKC-regulated factors, could lead to ectopic release of digestive enzymes into the interstitial space of the pancreas, resulting in organ injury. This is currently believed to be a contributing cause of clinical acute pancreatitis $(4,36,38)$. In fact, the morphologic events observed with supramaximal CCK stimulation of dispersed acini were identically observed in the wellestablished experimental pancreatitis model of supraphysiologic CCK stimulation of rodent pancreas (4-6, 36). We believe that the Munc18c displacement from the plasma membrane would render the membrane extremely receptive to pathologic exocytosis to occur. The parallel changes of actin disassembly $(30,31)$ would further allow free access of the ZGs to the acinar cell surface (56). Indeed, actin and associated cytoskeletal proteins have been postulated to be involved in physiologic (56) and pathologic exocytoses of the pancreatic acinar cell $(30,31)$. Additional cellular events might also be required for exocytosis to occur at the basolateral membrane surface. In particular, $\mathrm{Ca}^{2+}$ concentrations would have to be sufficiently high to consummate membrane fusion (57) at the basolateral membrane surface, which could indeed arise from the disruption of calcium signaling in acinar cells of cerulein-induced pancreatitis (58).

We therefore also postulate that this mechanism of basal membrane exocytosis is an underlying basis of pancreatitis. To our knowledge, this report represents the first demonstration of a link between SNARE protein dysregulation and pathologic fusion processes leading to acute pancreatitis. Since the digestive enzymes in the pancreatic interstitial tissue are believed to be a major factor determining the progression of mild to severe forms of hemorrhagic pancreatitis (59, 60), Munc18c and/or its regulation by PKC could be potential therapeutic targets for the design of novel drugs that might prevent this disease progression. Furthermore, this conceptual paradigm could be extended to explain other pathologic fusion processes in pancreatitis, particularly lysosome-ZG fusions $(6,15,37)$. Lysosomal SNARE proteins are now being identified (61). Additional research into these SNARE proteins and their interactions in the pancreatic acinar cell could lead to the full elucidation of the molecular mechanisms of these pathologic membrane fusion events occurring in pancreatitis (15).

\section{Acknowledgments}

Financial support for this work is provided by the Medical Research Council (MRC) of Canada (MT-13169), the Fraser Elliott Foundation, and the American Gastroenterology Association/Industry (Pharmacia and Upjohn) Research Scholarship to H.Y. Gaisano, and by the Deutsche Forschungsgemeinschaft (SFB 518-A5) to M.P. Lutz. A.M.F. Salapatek is an MRC/Banting and Best Diabetes Center Scholar. We also thank T. Nemoto and H. Kasai for their helpful discussion and assistance in the 3D-reconstructed images, Vay Liang Go for reviewing the manuscript, and Xiaohang Huang, Sandra Theimer, Sandra Kohler, and Hans Weidenbach for their technical assistance.

\footnotetext{
1. Palade, G. 1975. Intracellular aspects of the process of protein synthesis. Science. 189:347-358.

2. Beaudoin, A., and Grondin, G. 1991. Secretory pathways in animal cells: with emphasis on pancreatic acinar cells. J. Electron Microsc. 17:51-69.

3. Williams, J.A., and Yule, D.I. 1993. Stimulus-secretion coupling in pancreatic acinar cells. In The pancreas: biology, pathophysiology and disease. 2 nd edition. V.L.W. Go et al., editors. Raven Press. New York, New York, USA. 167-170.

4. Scheele, G., Adler, G., and Kern, H. 1987. Exocytosis occurs at the lateral plasma membrane of the pancreatic acinar cell during supramaximal secretagogue stimulation. Gastronenterology. 92:345-353.

5. Lampel, H., and Kern, H. 1977. Acute interstitial pancreatitis in the rat induced by excessive doses of a pancreatic secretagogue. Virchows Arch. 373:97-117.

6. Steer, M., and Meldolesi, J. 1987. The cell biology of experimental pancreatitis. N. Engl. J. Med. 317:144-150.

7. Klöppel, G., Dreyer, T., Willemer, S., Kern, H., and Adler, G. 1986. Human acute pancreatitis: its pathogenesis in the light of immunocytochemical and ultrastructural findings in acinar cells. Virchows Arch. 409:791-803.

8. Gaisano, H.Y., et al. 1989. Novel tool for the study of CCK-stimulated pancreatic enzyme secretion. J. Clin. Invest. 83:321-325.

9. Gaisano, H.Y., and Miller, L.J. 1992. Complex role of protein kinase C in mediating the supramaximal inhibition pancreatic secretion observed with cholecystokinin. Biochem. Biophys. Res. Commun. 187:488-506.

10. Gaisano, H.Y., Miller, L.J., and Foskett, J.K. 1994. Suppression of Ca2+ oscillations induced by cholecystokinin (CCK) and its analog OPE in rat pancreatic acinar cells by low-level protein kinase $\mathrm{C}$ activation without
} 
transition of the CCK receptor from a high- to low-affinity state. Pflugers Arch. 427:455-462.

11. Gaisano, H.Y., Wong, D., Sheu, L., and Foskett, J.K. 1994. Calcium release by cholecystokinin analogue OPE is $\mathrm{IP}_{3}$-dependent in single rat pancreatic acinar cells. Am. J. Physiol. 267:C220-C228.

12. Südhof, T.C. 1995. The synaptic vesicle cycle: a cascade of protein-protein interactions. Nature. 375:645-653.

13. Weber, T., et al. 1998. SNAREpins: minimal machinery for membrane fusion. Cell. 92:759-772.

14. Pevsner, J., Hsu, S.C., Braun, J.E.A., and Scheller, R.H. 1994. Specificity and regulation of a synaptic vesicle docking complex. Neuron. 13:353-361.

15. Gaisano, H.Y. 2000. A hypothesis: SNARE-ing the mechanisms of regulated exocytosis and pathologic membrane fusions in the pancreatic acinar cell. Pancreas. 20:217-226.

16. Fujita, Y., et al. 1996. Phosphorylation of Munc18/nSec1by protein kinase C. J. Biol. Chem. 271:2625-7628.

17. Shuang, R., et al. 1998. Regulation of Munc18/syntaxin 1A interaction by cyclin-dependent kinase $5(\mathrm{Cdk} 5)$ in nerve endings. J. Biol. Chem. 227:4958-4966.

18. Gaisano, H.Y., Sheu, L., Foskett, J.K., and Trimble, W.S. 1994. Tetanus toxin-light chain cleaves a vesicle-associated membrane protein (VAMP) isoform 2 in rat pancreatic zymogen granules and inhibits enzyme secretion. J. Biol. Chem. 269:17062-17066.

19. Gaisano, H.Y., et al. 1996. The VAMP family in pancreatic and parotid acinar cells. Gastroenterology. 111:1661-1669.

20. Gaisano, H.Y., Sheu, L., Wong, P.P.C., Klip, A., and Trimble, W.S. 1997. SNAP 23 is located in the basolateral plasma membrane of rat pancreatic acinar cells. FEBS Lett. 414:298-302.

21. Gaisano, H.Y., et al. 1996. Distinct locations of the syntaxin family of proteins in rat pancreatic acinar cells. Mol. Biol. Cell. 7:2019-2027.

22. Ravichandran, V., Chawla, A., and Roche, P.A. 1996. Identification of a novel syntaxin and VAMP binding protein, SNAP 23, expressed in nonneuronal tissues. J. Biol. Chem. 271:13300-13303.

23. Tellam, J.T., Mcintosh, S., and James, D.E. 1995. Molecular identification of two novel Munc18 isoforms expressed in non-neuronal tissues. J. Biol. Chem 270:5857-5863.

24. Tellam, J.T., et al. 1997. Characterization of Munc18c and syntaxin 4 in 3T3-L1 adipocytes. J. Biol. Chem. 272:6179-6186.

25. Araki, S., et al. 1997. Inhibition of the binding of SNAP 23 to syn-4 by Munc 18c. Biochem. Biophys. Res. Commun. 234:257-262.

26. Tamori, T., et al. 1998. Inhibition of insulin-induced GLUT4 translocation by Munc18c through interaction with syntaxin 4 in 3T3-L1 adipocytes. J. Biol. Chem. 273:19740-19746.

27. Angleson, J.K., Cochilla, A.J., Kilic, G., Nussinovitch, I., and Betz, W.J. 1999. Regulation of dense core release from neuroendocrine cells revealed by imaging of single exocytic events. Nat. Neurosci. 2:440-446.

28. Cochilla, A.J., Angleson, J.K., and Betz, W.J. 2000. Differential regulation of granule-to-granule and granule-to-plasma membrane fusion during secretion from rat pituitary lactotrophs. Regulation of dense core release from neuroendocrine cells revealed by imaging single exocytic events. $J$. Cell Biol. 150:839-848.

29. Gionvanucci, D.R., Yule, D.I., and Stuenkel, E.L. 1998. Optical measurement of stimulus-evoked membrane dynamics in single pancreatic acinar cells. Am. J. Physiol. 275:C732-C739.

30. Lerch, M.M., et al. 1997. Dissociation and reassembly of adherens junctions during experimental acute pancreatitis. Gastroenterology. 113:1355-1366.

31.Jungermann, J., Lerch, M.M., Weidenbach, H., Lutz, M.P., and Adler, G. 1995. Disassembly of rat pancreatic acinar cell cytoskeleton during supramaximal secretagogue stimulation. Am. J. Physiol. 268:G328-G338.

32. Valentijn, K.M., Gumkowski, F.D., and Jamieson, J.D. 1999. The subapical actin cytoskeleton regulates secretion and membrane retrieval in pancreatic acinar cells. J. Cell Sci. 112:81-96.

33. Arvan, P., and Castle, J.D. 1987. Phasic release of newly synthesized secretory proteins in the unstimulated rat exocrine pancreas. J. Cell Biol. 104:243-252.

34. Jena, B.P., et al. 1994. Redistribution of a Rab3-like GTP-binding protein from secretory granules to the Golgi complex in pancreatic acinar cells during regulated exocytosis. J. Cell Biol. 124:43-53.

35. Anderson, K.L., and McNiven, M.A. 1995. Vesicle dynamics during regulated secretion in a novel pancreatic acinar in vitro model. Eur. J. Cell Biol. 387:199-202.

36. Adler, G., Rohr, G., and Kern, H. 1982. Alteration of membrane fusion as a cause of acute pancreatitis in the rat. Dig. Dis. Sci. 27:993-1002.

37. Steer, M.L., and Saluja, A.K. 1993. Experimental acute pancreatitis: studies of the early events that lead to cell injury. In The pancreas: biology, pathobiology and disease. 2 nd edition. V.L.W. Go et al., editors. Raven Press. New York, New York, USA. 489-499.

38. Steinberg, W., and Tenner, S. 1994. Acute pancreatitis. N. Engl. J. Med. 330:1198-1210

39. Huang, X.H., Sheu, L., Trimble, W.S., and Gaisano, H.Y. 2001. CCK-regulated exocytosis in rat pancreatic acinar cells is inhibited by a C-terminus truncated mutant of SNAP-23. Pancreas. 23:125-133.

40. Hansen, N.J., Antonin, W., and Edwardson, J.M. 1999. Identification of SNAREs involved in regulated exocytosis in the pancreatic acinar cell. $J$. Biol. Chem. 274:22872-22876.

41. Guo, Z., Turner, C., and Castle, J.D. 1997. Relocation of the t-SNARE SNAP-23 from lamellipodia-like cell surface projections regulates compound exocytosis in mast cells. Cell. 94:537-548.

42. Olson, A.L., Knight, J.B., and Pessin, J.E. 1997. Syntaxin 4, VAMP-2, and/or VAMP-3 are functional t- and v-SNAREs for insulin-stimulated GLUT4 translocation in adipocytes. Mol. Cell. Biol. 17:2425-2435.

43. Thurmond, D.C., et al. 1998. Regulation of insulin stimulated GLUT4 translocation by Munc18c in 3T3L1 adipocytes. J. Biol. Chem. 273:33876-33883.

44. Kawanishi, M., et al. 2000. Role of SNAP23 in insulin induced translocation of GLUT4 in 3T3-L1 adipocytes. J. Biol. Chem. 275:8240-8247.

45. Jahn, R., and Sudhof, T.C. 1999. Membrane fusion and exocytosis. Annu. Rev. Biochem. 68:863-911.

46. Valentijn, J.A., Valentijn, K., Pastore, L.M., and Jamieson,.J.D. 2000. Actin coating of secretory granules during regulated exocytosis correlates with release of rab3D. Proc. Natl. Acad. Sci. USA. 97:1091-1095.

47. Ohnishi, H., Samuelson, L.C., Yule, D.I., Ernst, S.A., and Williams, J.A. 1997. Overexpression of Rab3D enhances regulated amylase secretion from pancreatic acini of transgenic mice. J. Clin. Invest. 100:3044-3052.

48. Reed, G.L., Huong, A.K., and Fitzgerald, M.L. 1999. Platelets contain SNARE proteins and a Sec1p homologue that interacts with syntaxin 4 and is phosphorylated after thrombin activation: implications for platelet secretion. Blood. 93:2617-2626

49. Schneider, S.W.K., Sritharan, K.C., Geibel, H., Oberleithner, H., and Jena, B.P. 1997. Surface dynamics in living acinar cells imaged by atomic force microscopy: identification of plasma membrane structures involved in exocytosis. Proc. Natl. Acad. Sci. USA. 94:316-321.

50. Nemoto, T., et al. 2001. Sequential-replenishment mechanism of exocytosis in pancreatic acini. Nat. Cell Biol. 3:253-258.

51. Grindstaff, K.K., et al. 1998. Sec6/8 complex is recruited to cell-cell contacts and specifies transport vesicle delivery to the basal-lateral membrane in epithelial cells. Cell. 93:731-740.

52. Dulubova, I., et al. 1999. A conformational switch in syntaxin during exocytosis: role of munc 18. EMBO J. 18:4372-4382.

53. Schulze, K.L., et al. 1994. Rop, a Drosophila homolog of yeast Sec1 and vertebrate n-Sec1/Munc18 proteins, is a negative regulator of neurotransmitter release in vivo. Neuron. 13:1099-1108.

54. Harrison, S.D., Broadie, K., van de Goor, J., and Rubin, G.M. 1994. Mutations in the Drosophila Rop gene suggest a function in general secretion and synaptic transmission. Neuron. 13:555-566.

55. Fisher, R.J., Pevsner, J., and Burgoyne, R.D. 2001. Control of fusion pore dynamics during exocytosis by Munc18. Science. 291:875-878.

56. Muallem, S., Kwiatkowska, K., Xu, X., and Yin, H. 1995. Actin filament disassembly is a sufficient final trigger for exocytosis in nonexcitable cells. J. Cell Biol. 128:589-598.

57. Tahara, M., Coorssen, J.R., Timmers, K., and Zimmerberg, J. 1998. Calcium can disrupt the SNARE protein complex on sea urchin egg secretory vesicles without irreversibly blocking fusion. J. Biol. Chem. 273:33667-33673.

58. Ward, J.B., Sutton, R., Jenkins, S.A., and Petersen, O.H. 1996. Progressive disruption of acinar cell calcium signaling is an early feature of ceruleininduced pancreatitis in mice. Gastroenterology. 111:481-491.

59. del Castillo, C.F., Schmide, J., Warshaw, A.L., and Rattner, D.W. 1994. Interstitial protease activation is the central event in progression to necrotizing pancreatitis. Surgery. 116:497-504.

60. Hartwig, W., et al. 1999. Interstitial trypsinogen release and its relevance to the transformation of mild into necrotizing pancreatitis in rats. Gastroenterology. 117:717-725.

61. Advani, R.J., et al. 1998. Seven novel mammalian SNARE proteins localize to distinct membrane compartments. J. Biol. Chem. 273:10317-10324. 